TRANSACTIONS OF THE

AMERICAN MATHEMATICAL SOCIETY

Volume 365, Number 3, March 2013, Pages 1637-1668

S 0002-9947(2012)05279-2

Article electronically published on July 25, 2012

\title{
ON THE $L$-FUNCTION OF MULTIPLICATIVE CHARACTER SUMS
}

\author{
JOHN DOLLARHIDE
}

\begin{abstract}
Let $\mathbb{F}_{q}$ be a finite field and let $\chi_{1}, \ldots, \chi_{r}$ be multiplicative characters on $\mathbb{F}_{q}$. Suppose there are homogeneous polynomials $f_{1}, \ldots, f_{r}$ of degrees $d_{1}, \ldots, d_{r}$ in $\mathbb{F}_{q}\left[x_{1}, \ldots, x_{n}\right]$ and suppose that $f_{1}, \ldots, f_{r}$ define smooth hypersurfaces in $\mathbb{P}^{n-1}$ that have normal crossings. When the character sum $S=\sum_{x \in \mathbb{P}^{n-1}\left(\mathbb{F}_{q}\right)} \chi_{1}\left(f_{1}(x)\right) \ldots \chi_{r}\left(f_{r}(x)\right)$ is well defined, we compute the $p$ adic Dwork cohomology of the $L$-function associated to $S$. In particular, we give a lower bound for the $p$-adic Newton polygon of the $L$-function and give a formula for the Hilbert series of the non-vanishing cohomology in terms of $n, r, q$ and the $d_{j}$ 's.
\end{abstract}

\section{INTRODUCTION}

Suppose $f_{1}, \ldots, f_{r}$ are homogeneous polynomials in $n$ variables with coefficients in $\mathbb{F}_{q}$. For each $j=1, \ldots, r$, let $\operatorname{deg} f_{j}=d_{j}$ and let $\chi_{j}: \mathbb{F}_{q}^{\times} \rightarrow \mathbb{C}_{p}^{\times}$be a multiplicative character on $\mathbb{F}_{q}$ such that $\prod_{j=1}^{r} \chi_{j}^{d_{j}}=\chi_{0}$, the trivial character. Define the character sum

$$
S(f, \chi)=S\left(f_{1}, \ldots, f_{r}, \chi_{1}, \ldots, \chi_{r}\right)=\sum_{x \in \mathbb{P}^{n-1}\left(\mathbb{F}_{q}\right)} \chi_{1}\left(f_{1}(x)\right) \ldots \chi_{r}\left(f_{r}(x)\right) .
$$

For each integer $m \geq 2$, we can define multiplicative characters $\chi_{1}^{(m)}, \ldots, \chi_{r}^{(m)}$ on $\mathbb{F}_{q^{m}}$, by composition of the characters $\chi_{j}$ with the norm map from $\mathbb{F}_{q^{m}}$ to $\mathbb{F}_{q}$, to get the associated sum

$$
S_{m}(f, \chi)=\sum_{x \in \mathbb{P}^{n-1}\left(\mathbb{F}_{q} m\right)} \chi_{1}^{(m)}\left(f_{1}(x)\right) \ldots \chi_{r}^{(m)}\left(f_{r}(x)\right) .
$$

These character sums generate the $L$-function

$$
L\left(f, \chi, \mathbb{P}^{n-1} ; t\right)=\exp \left(\sum_{m=1}^{\infty} S_{m}(f, \chi) t^{m} / m\right) .
$$

By a theorem of Katz [11, if $\left(p, d_{j}\right)=1$ for each $j$ and $\prod_{1}^{r} f_{j}=0$ defines a normal crossing divisor (each $f_{j}$ defines a smooth hypersurface in $\mathbb{P}^{n-1}$ and the normal vectors are linearly independent at any point where subsets of $\left\{f_{1}, \ldots, f_{r}\right\}$ intersect), then $L\left(f, \chi, \mathbb{P}^{n-1} ; t\right)^{(-1)^{n}}$ is a polynomial.

Received by the editors January 23, 2009 and, in revised form, August 28, 2011.

2010 Mathematics Subject Classification. Primary 11L40, 14F30.

Key words and phrases. Character sums, L-function, p-adic cohomology, Newton polygon. 
We also define the associated character sums in affine space

$$
S_{m}^{*}(f, \chi)=\sum_{x \in \mathbb{A}^{n}\left(\mathbb{F}_{q} m\right)} \chi_{1}^{(m)}\left(f_{1}(x)\right) \cdots \chi_{r}^{(m)}\left(f_{r}(x)\right)
$$

for all $m \geq 1$, with the relation $S_{m}^{*}(f, \chi)=\left(q^{m}-1\right) \cdot S_{m}(f, \chi)$. The $L$-functions are then related by the equation

$$
L\left(f, \chi, \mathbb{A}^{n} ; t\right)=\frac{L\left(f, \chi, \mathbb{P}^{n-1} ; q t\right)}{L\left(f, \chi, \mathbb{P}^{n-1} ; t\right)} .
$$

Take an additive character $\psi$ and let $\chi$ be a non-trivial multiplicative character. The Gauss sum associated to $\psi$ and $\chi$ is defined by $g(\chi, \psi)=\sum_{a \in \mathbb{F}_{q}} \chi(a) \psi(a)$. If $\chi_{j} \neq \chi_{0}$ for all $j=1, \ldots, r$, then

$$
\sum_{x \in \mathbb{F}_{q}^{n}} \chi_{1}\left(f_{1}(x)\right) \ldots \chi_{r}\left(f_{r}(x)\right)
$$

$$
=\left(\prod_{j=1}^{r} g\left(\chi_{j}^{-1}, \psi\right)\right)^{-1} \cdot \sum_{(x, y) \in \mathbb{F}_{q}^{n+r}} \chi_{1}^{-1}\left(y_{1}\right) \ldots \chi_{r}^{-1}\left(y_{r}\right) \cdot \psi\left(y_{1} f_{1}(x)+\cdots+y_{r} f_{r}(x)\right),
$$

where $y_{1}, \ldots, y_{r} \in \mathbb{F}_{q}^{\times}$. Therefore, up to the product of Gauss sums, it is equivalent to study sums of the form

$$
\sum_{(x, y) \in \mathbb{A}^{n+r}\left(\mathbb{F}_{q} m\right)} \chi_{1}^{(m)}\left(y_{1}\right) \ldots \chi_{r}^{(m)}\left(y_{r}\right) \cdot \psi^{(m)}\left(y_{1} f_{1}(x)+\cdots+y_{r} f_{r}(x)\right),
$$

where $\psi^{(m)}=\psi \circ \operatorname{Tr}_{\mathbb{F}_{q} m / \mathbb{F}_{q}}$. Accordingly, we define $T_{m}^{*}(f, \chi)$ as the sum

$$
\sum_{(x, y) \in \mathbb{A}^{n+r}\left(\mathbb{F}_{q}^{m}\right)}\left[\left(\chi_{1}^{-1}\right)^{(m)}\left(y_{1}\right) \ldots\left(\chi_{r}^{-1}\right)^{(m)}\left(y_{r}\right)\right] \cdot \psi^{(m)}\left(\sum_{j=1}^{r} y_{j} f_{j}(x)\right)
$$

and its associated $L$-function $L\left(f, \chi, \psi, T^{*} ; t\right)=\exp \left(\sum_{m=1}^{\infty} T_{m}^{*}(f, \chi) t^{m} / m\right)$.

In Chapter 2 we use Dwork's theory to relate this $L$-function to the characteristic polynomial of a Frobenius operator acting on a certain complex of differential forms. The Frobenius operator induces an action on the cohomology of the complex and in Chapter 3 we compute this cohomology (see Theorem 3.1).

In Chapter 4 we compute the Hilbert series associated to the non-vanishing cohomology space (equation (4-15)), and also generate a formula for the degree of the above $L$-function (see (4-19)). The dimensions that occur as coefficients of the Hilbert series are used in Chapter 5 to estimate a lower bound for the $p$-adic Newton polygon of the polynomial $L\left(f, \chi, \mathbb{P}^{n-1} ; t\right)$ (see Theorem 5.1).

Examples are contained in Chapter 6 for the values $n=1,2$ and 3 .

A brief history of the study of the sums of type (1-2) is given in [2]. Character sums of type (1-6) are studied in [4. In certain cases these sums can be used to count points on an associated variety and are therefore used to study the zeta function. For example, using the normal crossing divisor in $\mathbb{P}^{n-1}$ defined by $f_{1}(x, z)=x$, $f_{2}(x, z)=x-z, f_{3}(x, z)=x-\lambda z$ and $f_{4}(x, z)=z$ we can analyze the elliptic curve defined by the equation $y^{2}=(x)(x-1)(x-\lambda)$ for some $\lambda \neq 0,1$ in $\mathbb{F}_{q}$, as the number

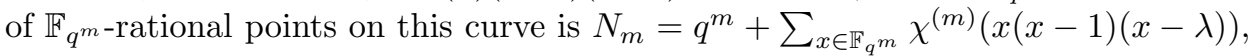


where $\chi^{(m)}$ is the quadratic character on $\mathbb{F}_{q^{m}}$. We note that in this particular case the cohomology can be computed explicitly and agrees with the formulas of Chapters 4 and 5 .

\section{The DWORK COMPleX}

Let $p$ be a prime number and let $q=p^{a}$ for any positive integer $a$. Denote the field of characteristic $p$ and cardinality $q$ by $\mathbb{F}_{q}$. The multiplicative norm map from $\mathbb{F}_{q}$ to $\mathbb{F}_{p}$ will be denoted by $\mathrm{N}_{\mathbb{F}_{q} / \mathbb{F}_{p}}$, and the additive trace map from $\mathbb{F}_{q}$ to $\mathbb{F}_{p}$ is denoted by $\operatorname{Tr}_{\mathbb{F}_{q} / \mathbb{F}_{p}}$. We let $\mathbb{Q}_{p}$ denote the field of $p$-adic numbers, with ring of integers $\mathbb{Z}_{p}$. The $p$-adic absolute value on $\mathbb{Q}_{p}$ will be denoted by $|\cdot|_{p}$ and the $p$-ordinal will be denoted by $\operatorname{ord}_{p}$.

Define $K=\mathbb{Q}_{p}\left(\zeta_{q-1}\right)$, the unramified extension of $\mathbb{Q}_{p}$ of degree $a$ with residue field $\mathbb{F}_{q}$. Let $\zeta_{p}$ denote a $p$ th root of unity and define $\Gamma_{1}=\mathbb{Q}_{p}\left(\zeta_{p}\right)$, a totally ramified extension of $\mathbb{Q}_{p}$ of degree $p-1$. If we take $\pi$ satisfying $\pi^{p-1}=-p$, then $\pi$ generates the maximal ideal in $\mathcal{O}_{\Gamma_{1}}$ with residue field $\mathcal{O}_{\Gamma_{1}} /(\pi) \mathcal{O}_{\Gamma_{1}}=\mathbb{F}_{p}$. We let $\Gamma_{0}=K(\pi)$. For extensions using fractional powers of $\pi$, we let $b$ be a positive rational number and write $\frac{(p-1) b}{q-1}=\frac{r_{1}}{r_{2}}$. Denote $\tilde{\pi}=\pi^{1 / r_{2}}$ and define the extensions $\widetilde{\Gamma}_{1}=\Gamma_{1}(\tilde{\pi})$ and $\widetilde{\Gamma}_{0}=\Gamma_{0}(\tilde{\pi})$.

We denote the Teichmüller character on $\mathbb{F}_{q}$ by $\widehat{\chi}$. The Teichmüller representative of the element of $\mathbb{F}_{q}^{\times}$with least positive residue $i$ will be denoted Teich $(i)$ or $\hat{i}$. On the other hand, the reduction of an element $x \in \mathbb{Q}_{p}\left(\zeta_{p-1}\right)^{\times}$to the corresponding element of $\mathbb{F}_{q}^{\times}$will be denoted $\bar{x}$. For a multiplicative character $\chi$ on $\mathbb{F}_{q}$ we can get a character on $\mathbb{F}_{q^{m}}$ by composing $\chi$ with the norm map from $\mathbb{F}_{q^{m}}$ to $\mathbb{F}_{q}$. In this case we will denote such a character by $\chi^{(m)}$. Similarly, for each $m>1$ we can get an additive character $\psi^{(m)}$ on $\mathbb{F}_{q^{m}}$ by composing the additive trace map $\operatorname{Tr}_{\mathbb{F}_{q^{m}} / \mathbb{F}_{q}}$ with an additive character $\psi$ on $\mathbb{F}_{q}$.

Consider the following infinite dimensional spaces of power series in several variables. We abbreviate a monomial in $n+r$ variables as

$$
A(u, v) x^{u} y^{v}=A\left(u_{1}, \ldots, u_{n}, v_{1}, \ldots, v_{r}\right) x_{1}^{u_{1}} \ldots x_{n}^{u_{n}} y_{1}^{v_{1}} \ldots y_{r}^{v_{r}},
$$

where $A(u, v) \in \widetilde{\Gamma}_{0}$. Let $|v|=\sum_{j=1}^{r} v_{j}$. For a positive rational number $b$ and a positive integer $d$ define

$$
C(b, d)=\left\{\sum_{u, v} A(u, v) \pi^{(p-1) b|v| / d} x^{u} y^{v / d}:|A(u, v)|_{p} \rightarrow 0 \text { as } u, v \rightarrow \infty\right\} .
$$

This space is complete with respect to the norm $\sup _{u, v}|A(u, v)|_{p}$ and we take as an orthonormal basis $\left\{\pi^{(p-1) b|v| / d} x^{u} y^{v / d}\right\}$.

We will actually work in some subspaces of $C(b, d)$ given by the following direct product decomposition. Let $\delta=\left(\delta_{1}, \ldots, \delta_{r}\right)$, with $0 \leq \delta_{j}<d$. For each such $\delta$ we define

$$
C(b, d, \delta)=\left\{\sum_{u, v} A(u, v) \pi^{(p-1) b|v| / d} x^{u} y^{v / d}: v_{j} \equiv \delta_{j} \quad(\bmod d)\right\} .
$$

It follows that $C(b, d)=\bigoplus_{\delta} C(b, d, \delta)$, a product of $d^{r}$ subspaces. The $r$-tuple $\delta$ will be associated to the orginal multiplicative characters in the following way. If $\hat{\chi}(z)=\hat{z}$, then we define $\delta_{j}$ using the relation $\chi_{j}=\widehat{\chi}^{\delta_{j}}$, for each $j=1, \ldots, r$. 
Using the splitting functions of Dwork, we use certain power series to construct a lifting of characters from characteristic $p$ to characteristic zero. The Artin-Hasse exponential function is given by the series $E(t)=\exp \left(\sum_{j=0}^{\infty} \frac{t^{p^{j}}}{p^{j}}\right)$ and converges for all $|t|_{p}<1$. It has $p-1$ zeros with $p$-ordinal $\frac{1}{p-1}$. We choose one and call it $\gamma$. Now define the series $\Theta(t)=E(\gamma t)$. This is a splitting function as described by Dwork [8]. Writing as a power series $\Theta(t)=\sum_{i=0}^{\infty} \lambda_{i} t^{i}$, it can be shown that $\lambda_{0}=1$ and $\gamma^{i} \mid \lambda_{i}$ for each $i$, so that $\operatorname{ord}_{p}\left(\lambda_{i}\right) \geq \frac{i}{(p-1)}$. Since $\Theta(1)^{p}=1$ and since $\Theta(1) \equiv 1+\gamma\left(\bmod \gamma^{2}\right)$ in $\mathcal{O}_{\Gamma_{1}}$ it follows that $\Theta(1)$ is a primitive $p$ th root of unity.

Writing $f_{j}=\sum_{u \in \mathcal{U}_{j}} a_{u, j} x^{u}$ we define the series

$$
G(x, y)=\prod_{\substack{u \in \mathcal{U}_{j} \\ 1 \leq j \leq r}} \Theta\left(\hat{a}_{u, j} x^{u} y_{j}\right) \quad \text { and } \quad H(x, y)=\prod_{k=0}^{a-1} G\left(x^{p^{k}}, y^{p^{k}}\right) .
$$

It can be shown that $G \in C(b, 1)$ for all $b<1 /(p-1)$ and $H \in C(b, 1)$ for all $b<p /(q(p-1))$. Also multiplication by $H$ is stable on $C(b / q, d, \delta)$ for each $\delta$ and it is bounded on bounded subsets of $C(b, d)$, hence continuous.

Now consider the inclusion map $C(p b, d) \rightarrow C(b, d)$. The associated diagonal matrix has entries $\left(\pi^{p b-b}, \pi^{2 p b-2 b}, \pi^{3 p b-3 b}, \ldots\right)$ and is therefore completely continuous in the sense of 17 .

If $\delta$ is the least non-negative residue of $p \delta^{\prime}$ modulo $d$, then for any element $\xi=\sum_{u, v} A(u, v) x^{u} y^{v / d}$ in $C(b, d, \delta)$ we define the map $\Psi: C(b, d, \delta) \rightarrow C\left(p b, d, \delta^{\prime}\right)$ by the formula

$$
\Psi(\xi)=\sum_{u, v} A(p u, p v) x^{u} y^{v / d} .
$$

We now define the Frobenius morphism $\alpha=\Psi^{a} \circ H$ as the composition

$$
C(b, d, \delta) \stackrel{i}{\longrightarrow} C(b / q, d, \delta) \stackrel{H}{\longrightarrow} C(b / q, d, \delta) \stackrel{\Psi^{a}}{\longrightarrow} C(b, d, \delta),
$$

where the map on the left is inclusion and the map in the center is multiplication by $H$. From the definition of $\Theta$ and since $G(x, y) \circ \Psi=\Psi \circ G\left(x^{p}, y^{p}\right)$, if $d \mid(q-1)$ it follows that $(\Psi \circ G)^{a}=\Psi^{a} \circ H$. Since $\Psi$ is also continuous on $C(b, d)$, the composition $\alpha=\Psi^{a} \circ H$ is a completely continuous endomorphism of $C(b, d, \delta)$ for all $b<p /(p-1)$.

We define the related operator $\beta$, an ath root of $\alpha$, for which $p$-adic information on the coefficients is much easier to obtain. Let $\tau \in \operatorname{Gal}\left(\Gamma_{0} / \Gamma_{1}\right)$ such that $\tau(\pi)=\pi$ and let $\beta=\Psi \circ \tau^{-1} \circ G$, which satisfies $\beta^{a}=\alpha$.

The Dwork trace formula gives an equality between the trace of $\alpha$ and twisted exponential sums.

Theorem 2.1 (Dwork Trace Formula). For any $m \geq 1$,

$$
\begin{aligned}
& \left(q^{m}-1\right)^{n+r} \operatorname{Tr}\left(\alpha^{m} \mid C(b, d, \delta)\right) \\
& =\sum_{(x, y) \in \mathbb{F}_{q^{m}}^{n+r}}\left(\hat{\chi}^{(m)}\left(y_{1}\right)\right)^{-\frac{(q-1) \delta_{1}}{d}} \ldots\left(\hat{\chi}^{(m)}\left(y_{r}\right)\right)^{-\frac{(q-1) \delta_{r}}{d}} \cdot H(\hat{x}, \hat{y}) .
\end{aligned}
$$

Proof. See [1].

Moreover, if $d=q-1$, then (2-5) is equal to the sum $T_{m}^{*}(f, \chi)$ in (1-6). 
From [17, the classification of the operator $\alpha$ as completely continuous allows the application of

$$
\operatorname{det}(I-t M)=\exp \left(-\sum_{j=1}^{\infty} \operatorname{Tr}\left(M^{j}\right) t^{j} / j\right)
$$

This determinant is a $p$-adic entire function.

We can analyze $\alpha$ further by extending its action onto a de Rham-type complex of differential forms with the space $C(b, d, \delta)$ as coefficients. Let

$$
\Omega_{C(b, d, \delta)}^{k}\langle Y\rangle=\bigoplus_{\ell+m=k} \bigoplus_{\substack{1 \leq i_{1}<\cdots<i_{\ell} \leq n \\ 1 \leq j_{1}<\cdots<j_{m} \leq r}} C(b, d, \delta) d x_{i_{1}} \wedge \cdots \wedge d x_{i_{\ell}} \wedge \frac{d y_{j_{1}}}{y_{j_{1}}} \wedge \cdots \wedge \frac{d y_{j_{m}}}{y_{j_{m}}}
$$

For some $g \in C(b, d, \delta)$ define $d_{\langle Y\rangle}: \Omega_{C(b, d, \delta)}^{k}\langle Y\rangle \longrightarrow \Omega_{C(b, d, \delta)}^{k+1}\langle Y\rangle$ by (dropping $\wedge$ from the notation)

$$
\begin{aligned}
d_{\langle Y\rangle} & \left(g d x_{i_{1}} \ldots d x_{i_{\ell}} \frac{d y_{j_{1}}}{y_{j_{1}}} \ldots \frac{d y_{j_{m}}}{y_{j_{m}}}\right) \\
= & \left(\sum_{i=1}^{n} \frac{\partial g}{\partial x_{i}} d x_{i}+\sum_{j=1}^{r} y_{j} \frac{\partial g}{\partial y_{j}} \frac{d y_{j}}{y_{j}}\right) d x_{i_{1}} \ldots d x_{i_{\ell}} \frac{d y_{j_{1}}}{y_{j_{1}}} \ldots \frac{d y_{j_{m}}}{y_{j_{m}}} .
\end{aligned}
$$

Using $d_{\langle Y\rangle}$ we now define a homomorphism $\Delta: \Omega_{C(b, d, \delta)}^{k}\langle Y\rangle \rightarrow \Omega_{C(b, d, \delta)}^{k+1}\langle Y\rangle$ as follows. For an integer $s>0$ let $\gamma_{s}=\sum_{i=0}^{s} \gamma^{p^{i}} / p^{i}$ and for $\widehat{F}$, the Teichmüller lifting of $F=\sum_{j=1}^{r} y_{j} f_{j}(x)$, we set

$$
\begin{aligned}
\widetilde{F}(x, y) & =\sum_{s=0}^{\infty} \gamma_{s} \widehat{F}^{\tau^{s}}\left(x^{p^{s}}, y^{p^{s}}\right) \\
& =\sum_{s=0}^{\infty} \gamma_{s} \sum_{j=1}^{r} y_{j}^{p^{s}} \hat{f}_{j}^{\tau^{s}}\left(x^{p^{s}}\right),
\end{aligned}
$$

which is an element of $C(b, 1)$. It follows that $d_{\langle Y\rangle}(\widetilde{F}) \in \Omega_{C(b, 1)}^{1}\langle Y\rangle$. Now let $\Delta: \Omega_{C(b, d, \delta)}^{k}\langle Y\rangle \longrightarrow \Omega_{C(b, d, \delta)}^{k+1}\langle Y\rangle$ be given by

$$
\Delta(\omega)=d_{\langle Y\rangle}(\omega)+d_{\langle Y\rangle}(\widetilde{F}) \wedge \omega
$$

which yields the complex

$$
\left(\Omega_{C(b, d, \delta)}^{\bullet}\langle Y\rangle, \Delta\right)
$$

Let $g \in C(b, d, \delta)$. Extending the Frobenius morphism $\alpha$ we can define a map of complexes $\alpha_{\bullet}: \Omega_{C(b, d, \delta)}^{\bullet}\langle Y\rangle \longrightarrow \Omega_{C(b, d, \delta)}^{\bullet}\langle Y\rangle$ by linearity on $C(b, d, \delta)$ and the formula

$$
\begin{aligned}
& \alpha_{k}\left(g d x_{i_{1}} \ldots d x_{i_{\ell}} \frac{d y_{j_{1}}}{y_{j_{1}}} \ldots \frac{d y_{j_{m}}}{y_{j_{m}}}\right) \\
& \quad=\frac{q^{n+r-k}}{x_{i_{1}} \ldots x_{i_{\ell}}} \alpha\left(x_{i_{1}} \ldots x_{i_{\ell}} g\right) d x_{i_{1}} \ldots d x_{i_{\ell}} \frac{d y_{j_{1}}}{y_{j_{1}}} \ldots \frac{d y_{j_{m}}}{y_{j_{m}}} .
\end{aligned}
$$


A straightforward computation shows that $\Delta \circ \alpha=\alpha \circ \Delta$ so that $\alpha_{\bullet}$ is a chain map on the complex (2-10). Also note that $\beta$ induces a similar map of complexes given by

$$
\begin{aligned}
& \beta_{k}\left(g d x_{i_{1}} \ldots d x_{i_{\ell}} \frac{d y_{j_{1}}}{y_{j_{1}}} \ldots \frac{d y_{j_{m}}}{y_{j_{m}}}\right) \\
& \quad=\frac{p^{n+r-k}}{x_{i_{1}} \ldots x_{i_{\ell}}} \beta\left(x_{i_{1}} \ldots x_{i_{\ell}} g\right) d x_{i_{1}} \ldots d x_{i_{\ell}} \frac{d y_{j_{1}}}{y_{j_{1}}} \ldots \frac{d y_{j_{m}}}{y_{j_{m}}} .
\end{aligned}
$$

Now since $\alpha$ is a chain map, it will induce a map on the cohomology of the complex (2-10). Moreover from [17, since each morphism $\alpha_{k}$ is completely continuous we have the relation

$$
\sum_{k=0}^{n+r}(-1)^{k} \operatorname{Tr}\left(\alpha_{k} \mid \Omega_{C(b, d, \delta)}^{k}\langle Y\rangle\right)=\sum_{k=0}^{n+r}(-1)^{k} \operatorname{Tr}\left(\alpha_{k} \mid H^{k}\left(\Omega_{C(b, d, \delta)}^{\bullet}\langle Y\rangle\right)\right) .
$$

Combining the above with Theorem 2.1 we can apply the following version of the trace formula due to Robba [15]:

$$
L\left(f, \chi, \psi, T^{*} ; t\right)=\prod_{k=0}^{n+r} \operatorname{det}\left(I-t \alpha_{k} \mid H^{k}\left(\Omega_{C(b, d, \delta)}^{\bullet}\langle Y\rangle, \Delta\right)\right)^{(-1)^{k+1}} .
$$

\section{Computation of Cohomology}

Before analyzing the action of $\alpha$ and the determinants in (2-14), we compute the cohomology of the Dwork complex. In this chapter we prove the following.

Theorem 3.1. Suppose $f_{1}, \ldots, f_{r}$ define a divisor with normal crossings with degrees satisfying $\left(p, d_{j}\right)=1$. Then $H^{k}\left(\Omega_{C(b, d, \delta)}^{\bullet}\langle Y\rangle, \Delta\right)=0$ for $0 \leq k \leq n+r-2$ and $H^{n+r-1}\left(\Omega_{C(b, d, \delta)}^{\bullet}\langle Y\rangle, \Delta\right) \cong H^{n+r}\left(\Omega_{C(b, d, \delta)}^{\bullet}\langle Y\rangle, \Delta\right)$.

3.1. Reduction to characteristic $p$. We first simplify the computation, with a reduction, using the fact that the operator $\alpha$ is built from homogeneous elements. Define a grading on $\widetilde{\Gamma}_{0}[x, y]$ by

$$
\operatorname{deg}_{1} x_{i}=1, \text { for } i=1, \ldots, n \text { and } \operatorname{deg}_{1} y_{j}=-d_{j}, \text { for } j=1, \ldots, r \text {. }
$$

Let $\widehat{F}$ be the Teichmüller lifting of $F(x, y)=\sum_{j=1}^{r} y_{j} f_{j}(x)$, which will be a polynomial in $\mathcal{O}_{\Gamma_{0}}[x, y]$. We can see that all of the monomials in $\widehat{F}$ satisfy $\operatorname{deg}_{1}=0$. Therefore $G$ and $H$ defined in (2-2), satisfy $\operatorname{deg}_{1}=0$. Denote the subspace of $C(b, d, \delta)$ consisting of elements having $\operatorname{deg}_{1}=0$ by $C(b, d, \delta)^{(0)}$. It can be shown that for $d=q-1$ and for each acceptable choice of $b$ and $\delta$, that $\operatorname{Tr}(\alpha \mid C(b, q-1, \delta))=$ $\operatorname{Tr}\left(\alpha \mid C(b, q-1, \delta)^{(0)}\right)$.

By defining

$$
\operatorname{deg}_{1} d x_{i}=1 \quad \text { and } \quad \operatorname{deg}_{1} d y_{j}=-d_{j},
$$

we can extend this grading to $\Omega_{C(b, q-1, \delta)}^{\bullet}\langle Y\rangle$, where we denote the subspace of forms satisfying $\operatorname{deg}_{1}=0$ by $\Omega_{C(b, q-1, \delta)}^{\bullet}\langle Y\rangle^{(0)}$. We have $\alpha_{k}\left(\Omega_{C(b, q-1, \delta)}^{k}\langle Y\rangle^{(0)}\right) \subseteq$ $\Omega_{C(b, q-1, \delta)}^{k}\langle Y\rangle^{(0)}$ and moreover $\Delta\left(\Omega_{C(b, q-1, \delta)}^{k}\langle Y\rangle^{(0)}\right) \subseteq \Omega_{C(b, q-1, \delta)}^{k+1}\langle Y\rangle^{(0)}$, so that $\Omega_{C(b, q-1, \delta)}^{\bullet}\langle Y\rangle^{(0)}$ is a subcomplex of $\Omega_{C(b, q-1, \delta)}^{\bullet}\langle Y\rangle$ satisfying the condition $\operatorname{det}\left(I-t \alpha_{k} \mid \Omega_{C(b, q-1, \delta)}^{k}\langle Y\rangle / \Omega_{C(b, q-1, \delta)}^{k}\langle Y\rangle^{(0)}\right)=1$. Therefore we can reduce the computation of cohomology to that of the subcomplex $\left(\Omega_{C(b, q-1, \delta)}^{\bullet}\langle Y\rangle^{(0)}, \Delta\right)$. 
The space $C(b, q-1, \delta)$ has a natural decreasing filtration by $s \in \mathbb{Z}$ given by coefficients in the principal ideals $\tilde{\pi}^{s} \mathcal{O}_{\tilde{\Gamma}_{0}}$. Let

$$
\begin{aligned}
\mathcal{F}^{s} C & (b, q-1, \delta) \\
= & \left\{\sum_{u, v} A(u, v) \pi^{(p-1) b|v| /(q-1)} x^{u} y^{v /(q-1)} \mid A(u, v) \in \tilde{\pi}^{s} \mathcal{O}_{\widetilde{\Gamma}_{0}} \text { for all }(u, v)\right\} .
\end{aligned}
$$

We can extend this filtration to the Dwork complex by defining

$$
\begin{aligned}
\mathcal{F}^{s} \Omega_{C(b, q-1, \delta)}^{k}\langle Y\rangle & =\bigoplus_{\ell+m=k} \bigoplus_{\substack{1 \leq i_{1}<\cdots<i_{\ell} \leq n \\
1 \leq j_{1}<\cdots<j_{m} \leq r}} \mathcal{F}^{s} C(b, q-1, \delta) d x_{i_{1}} \ldots d x_{i_{\ell}} \frac{d y_{j_{1}}}{y_{j_{1}}} \ldots \frac{d y_{j_{m}}}{y_{j_{m}}} .
\end{aligned}
$$

Let $\Omega_{\mathbb{F}_{q}[x, y] / \mathbb{F}_{q}}\langle Y\rangle^{(0)}$ denote the algebra of differential forms (logarithmic in the $y$ 's) with coefficients in the polynomial ring $\mathbb{F}_{q}[x, y]$ and with all terms having $\operatorname{deg}_{1}=0$. If $\bar{A}(u, v)$ is the reduction of $A(u, v)$ modulo the ideal $\tilde{\pi} \mathcal{O}_{\tilde{\Gamma}_{0}}$, we can define a homomorphism of modules from $\mathcal{F}^{0} \Omega_{C(b, q-1, \delta)}^{k}\langle Y\rangle^{(0)}$ to $\Omega_{\mathbb{F}_{q}[x, y] / \mathbb{F}_{q}}^{k}\langle Y\rangle^{(0)}$ by

$$
\begin{aligned}
& \sum_{u, v} A(u, v) \pi^{(p-1) b|v| /(q-1)} x^{u} y^{v /(q-1)} d x_{i_{1}} \ldots d x_{i_{\ell}} \frac{d y_{j_{1}}}{y_{j_{1}}} \ldots \frac{d y_{j_{m}}}{y_{j_{m}}} \\
& \mapsto \sum_{u, v} \bar{A}(u, v) x^{u} y^{v /(q-1)} d x_{i_{1}} \ldots d x_{i_{\ell}} \frac{d y_{j_{1}}}{y_{j_{1}}} \ldots \frac{d y_{j_{m}}}{y_{j_{m}}} .
\end{aligned}
$$

The kernel of this homomorphism is $\mathcal{F}^{1} \Omega_{C(b, q-1, \delta)}^{k}\langle Y\rangle^{(0)}$ and therefore we have an isomorphism

$$
\mathcal{F}^{0} \Omega_{C(b, q-1, \delta)}^{k}\langle Y\rangle^{(0)} / \mathcal{F}^{1} \Omega_{C(b, q-1, \delta)}^{k}\langle Y\rangle^{(0)} \cong \Omega_{\mathbb{F}_{q}[x, y] / \mathbb{F}_{q}}^{k}\langle Y\rangle^{(0)} .
$$

Under this reduction, $\pi^{(p-1) b} \gamma^{-1} \Delta \equiv d_{\langle Y\rangle} F \wedge\left(\bmod \mathcal{F}^{1} \Omega_{C(b, q-1, \delta)}^{k}\langle Y\rangle^{(0)}\right)$, so that we have an isomorphism of complexes:

$$
\begin{gathered}
\left(\mathcal{F}^{0} \Omega_{C(b, q-1, \delta)}^{k}\langle Y\rangle^{(0)} / \mathcal{F}^{1} \Omega_{C(b, q-1, \delta)}^{k}\langle Y\rangle^{(0)}, \pi^{(p-1) b} \gamma^{-1} \Delta\right) \\
\cong\left(\Omega_{\mathbb{F}_{q}[x, y] / \mathbb{F}_{q}}^{k}\langle Y\rangle^{(0)}, d_{\langle Y\rangle}(F) \wedge\right) .
\end{gathered}
$$

It will suffice to compute the cohomology of this associated graded complex (see Section 3.5).

Now, since each $v_{j}$ satisfies $v_{j} \equiv \delta_{j}(\bmod q-1)$, we can work with integer powers of the $y$ 's if we remove a factor of $y_{1}^{\delta_{1} /(q-1)} \cdot \ldots \cdot y_{r}^{\delta_{r} /(q-1)}$ and work over the space $\mathbb{F}_{q}[x, y]^{(D)}$, where $D=\frac{1}{q-1} \sum_{j=1}^{r} d_{j} \delta_{j} \in \mathbb{N}$. For the rest of this chapter we will remove this factor and work on the corresponding spaces $\Omega_{\mathbb{F}_{q}[x, y] / \mathbb{F}_{q}}^{k}\langle Y\rangle^{(D)}$ without affecting the action of $\alpha$ on cohomology.

We write (3-7), adjusted for $D$, as a double complex

$$
\Omega_{\mathbb{F}_{q}[x, y] / \mathbb{F}_{q}}^{k}\langle Y\rangle^{(D)}=\bigoplus_{\ell+m=k}\left(\boldsymbol{\Omega}^{(\ell, m)}\right)^{(D)},
$$


where

$$
\left(\boldsymbol{\Omega}^{(\ell, m)}\right)^{(D)}=\underset{\substack{1 \leq i_{1}<\cdots<i_{\ell} \leq n \\ 1 \leq j_{1}<\cdots<j_{m} \leq r}}{\bigoplus} \mathbb{F}_{q}[x, y]^{(D-\ell)} d x_{i_{1}} \ldots d x_{i_{\ell}} \frac{d y_{j_{1}}}{y_{j_{1}}} \ldots \frac{d y_{j_{m}}}{y_{j_{m}}} .
$$

In this double complex there are $n+1$ columns indexed by $\ell$ and $r+1$ rows indexed by $m$ and the orginal complex is recovered by summing down the diagonals. The operator $d_{\langle Y\rangle}(F)$ can be divided into components $\partial_{v}=\sum_{j=1}^{r} f_{j} d y_{j}=\sum_{j=1}^{r} y_{j} f_{j} d y_{j} / y_{j}$ and $\partial_{h}=\sum_{j=1}^{r} y_{j} d f_{j}$, which satisfy the identity $\partial_{v} \partial_{h}+\partial_{h} \partial_{v}=0$.

We first consider the cohomology of each of the columns.

Proposition 3.1. For each $\ell=0, \ldots, n$, we have $H^{k}\left(\left(\boldsymbol{\Omega}^{(\ell, \bullet)}\right)^{(D)}, \partial_{v}\right)=0$ for $k<r$.

Proof. To verify the elements $\left\{y_{j} f_{j}\right\}_{j=1}^{r}$ form a regular sequence on $\mathbb{F}_{q}[x, y]^{(D-\ell)}$, we check that the following multiplication is injective for each $k=1, \ldots, r$ :

$$
\mathbb{F}_{q}[x, y]^{(D-\ell)} / \sum_{j=1}^{k-1} y_{j} f_{j} \mathbb{F}_{q}[x, y]^{(D-\ell)} \stackrel{y_{k} f_{k}}{\longrightarrow} \mathbb{F}_{q}[x, y]^{(D-\ell)} / \sum_{j=1}^{k-1} y_{j} f_{j} \mathbb{F}_{q}[x, y]^{(D-\ell)} \text {. }
$$

Separating out the powers of the $y_{j}$ 's we can write

$$
\mathbb{F}_{q}[x, y]^{(D-\ell)} / \sum_{j=1}^{k-1} y_{j} f_{j} \mathbb{F}_{q}[x, y]^{(D-\ell)}=\bigoplus_{v_{1}, \ldots, v_{r} \geq 0} y_{1}^{v_{1}} \ldots y_{r}^{v_{r}}\left[\mathbb{F}_{q}[x] / I_{v}\right]^{\left(D-\ell+\sum v_{j} d_{j}\right)},
$$

where $I_{v}=I\left(v_{1}, \ldots, v_{r}\right)$ is the ideal of $\mathbb{F}_{q}[x]$ generated by the $f_{i}$ for which $1 \leq i \leq$ $k-1$ and $v_{i}$ is strictly greater than zero.

Suppose first that the set $\left\{i: 1 \leq i \leq k-1\right.$ and $\left.v_{i}>0\right\}$ has cardinality $<n$. It follows that $\left\{f_{i}: 1 \leq i \leq k-1\right.$ and $\left.v_{i}>0\right\} \cup\left\{f_{k}\right\}$ form a regular sequence on $\mathbb{F}_{q}[x]$ by the normal crossing divisor hypothesis on $f_{1}, \ldots, f_{r}$. Therefore the multiplication in (3-9) is injective.

On the other hand suppose the set $\left\{i: 1 \leq i \leq k-1\right.$ and $\left.v_{i}>0\right\}$ has cardinality $\geq n$. Without loss of generality, we can assume that $\{1, \ldots, n\}$ is in this set. The normal crossing divisor hypothesis on $f_{1}, \ldots, f_{r}$ implies that $f_{1}, \ldots, f_{n}$ form a regular sequence in $\mathbb{F}_{q}\left[x_{1}, \ldots, x_{n}\right]$. Since the Hilbert series for the quotient space $\mathbb{F}_{q}[x] /\left(f_{1}, \ldots, f_{n}\right)$ is given by $\prod_{i=1}^{n}\left(1+t+t^{2}+\cdots+t^{d_{i}-1}\right)$, which is a polynomial of degree $d_{1}+\cdots+d_{n}-n$, it follows that in dimensions $k>\sum_{j=1}^{n} d_{j}-n$, the quotient $\left[\mathbb{F}_{q}[x] /\left(f_{1}, \ldots, f_{n}\right)\right]^{(k)}$ is zero. Therefore the multiplication is trivially injective.

3.2. Vanishing theorems and division lemmas for differential forms. In this section we compile various results from commutative algebra involving the vanishing of differential forms which are used in the computation of the cohomology along the top horizontal row of the double complex $\left(\boldsymbol{\Omega}^{(\bullet, \bullet)}\right)^{(D)}$.

For fixed elements $\omega_{1}, \ldots, \omega_{s} \in \Omega_{\mathbb{F}_{q}[x] / \mathbb{F}_{q}}^{1}$ write

$$
\omega_{1} \wedge \cdots \wedge \omega_{s}=\sum_{1 \leq i_{1}<\cdots<i_{s} \leq n} g_{i_{1}, \ldots, i_{s}} d x_{i_{1}} \wedge \cdots \wedge d x_{i_{s}},
$$

and let $I$ denote the ideal of $\mathbb{F}_{q}[x]$ generated by the $g_{i_{1}, \ldots, i_{s}}$.

We first recall the Saito-de Rham division lemma.

Proposition 3.2. Suppose $\omega \in \Omega_{\mathbb{F}_{q}[x] / \mathbb{F}_{q}}^{k}$ such that $\omega_{1} \wedge \cdots \wedge \omega_{s} \wedge \omega=0$. 
(a) There exists an integer $M \geq 0$ such that if $g \in I^{M}$, then

$$
g \omega=\sum_{j=1}^{s} \omega_{j} \wedge \varphi_{j}
$$

for some $\varphi_{1}, \ldots, \varphi_{s} \in \Omega_{\mathbb{F}_{q}[x] / \mathbb{F}_{q}}^{k-1}$.

(b) If $k<\operatorname{depth}(I)$, then there exist $\varphi_{1}, \ldots, \varphi_{s} \in \Omega_{\mathbb{F}_{q}[x] / \mathbb{F}_{q}}^{k-1}$ such that

$$
\omega=\sum_{j=1}^{s} \omega_{j} \wedge \varphi_{j} .
$$

Proof. See the main theorem of [16].

The next result is a generalization of Proposition 3.2 due to Adolphson and Sperber.

Proposition 3.3. Let $\omega \in \Omega_{\mathbb{F}_{q}[x] / \mathbb{F}_{q}}^{k}$ satisfy $\omega_{j} \wedge \omega=0$ for each $j=1, \ldots, s$.

(a) There exists an integer $M \geq 0$ with the property that if $g \in I^{M}$, then

$$
g \omega=\omega_{1} \wedge \cdots \wedge \omega_{s} \wedge \varphi
$$

for some $\varphi \in \Omega_{\mathbb{F}_{q}[x] / \mathbb{F}_{q}}^{k-s}$. that

(b) If $\operatorname{depth}(I)>0$ and $k<\operatorname{depth}(I)+s-1$, then there exists $\varphi \in \Omega_{\mathbb{F}_{q}[x] / \mathbb{F}_{q}}^{k-s}$ such

$$
\omega=\omega_{1} \wedge \cdots \wedge \omega_{s} \wedge \varphi
$$

Proof. See [5], Proposition 2.2.

Now consider the spaces

$$
\begin{aligned}
\widetilde{\Omega}^{k} & =\mathbb{F}_{q}\left[y_{1}, \ldots, y_{s}\right] \otimes \Omega_{\mathbb{F}_{q}[x] / \mathbb{F}_{q}}^{k} \\
& =\bigoplus_{v_{1}, \ldots, v_{s} \geq 0} y_{1}^{v_{1}} \ldots y_{s}^{v_{s}} \Omega_{\mathbb{F}_{q}[x] / \mathbb{F}_{q}}^{k}
\end{aligned}
$$

and the subspaces

$$
\left(\widetilde{\Omega}^{k}\right)^{(J)}=\bigoplus_{v_{1}+\cdots+v_{s}=J} y_{1}^{v_{1}} \ldots y_{s}^{v_{s}} \Omega_{\mathbb{F}_{q}[x] / \mathbb{F}_{q}}^{k}
$$

In [5], Proposition 3.2 and Proposition 3.3 are used to deduce the following:

Proposition 3.4. For $\omega_{1}, \ldots, \omega_{s}$ and $I$ as before and $\partial(\omega)=\left(y_{1} \omega_{1}+\cdots+y_{s} \omega_{s}\right) \wedge \omega$ :

(a) For each $J \geq 1$ and $k \geq 0$, there exists $M \geq 0$ such that

$$
I^{M} H^{k}\left(\left(\widetilde{\Omega}^{\bullet}\right)^{(J)}, \partial\right)=0 .
$$

(b) If $1 \leq J<\operatorname{depth}(I)$ and $k<\operatorname{depth}(I)+s-1$, then

$$
H^{k}\left(\left(\widetilde{\Omega}^{\bullet}\right)^{(J)}, \partial\right)=0 .
$$

Proof. See [5], Proposition 2.10.

The last few results involve the vanishing of logarithmic forms. 
Proposition 3.5. Suppose that $h_{1}, \ldots, h_{s}$ are homogeneous polynomials in $\mathbb{F}_{q}[x]=$ $\mathbb{F}_{q}\left[x_{1}, \ldots, x_{n}\right]$ that define a divisor with normal crossings. Let $t$ be an integer such that $t+k \leq n-1$ and let $\eta \in \Omega_{\mathbb{F}_{q}[x] / \mathbb{F}_{q}}^{k}$ such that

$$
d h_{j_{1}} \wedge \cdots \wedge d h_{j_{t}} \wedge \eta \equiv 0 \quad\left(\bmod h_{j_{1}}, \ldots, h_{j_{t}}\right) .
$$

Then

$$
\eta \equiv 0 \quad\left(\bmod d h_{j_{1}}, \ldots, d h_{j_{t}}, h_{j_{1}}, \ldots, h_{j_{t}}\right) .
$$

Proof. See [12, Lemma 2.2.2.

Proposition 3.5 is the main tool used to prove the following vanishing theorem.

Proposition 3.6. Suppose $h_{1}, \ldots, h_{s}$ are non-constant, homogeneous polynomials defining a normal crossing divisor. Let $0 \leq k \leq n-2$ and suppose $\eta \in \Omega_{\mathbb{F}_{q}[x] / \mathbb{F}_{q}}^{k}$ such that $d h_{j} \wedge \eta \equiv 0\left(\bmod h_{j}\right)$ for each $j=1, \ldots, s$. Then $\eta$ can be written as

$$
\begin{aligned}
\eta= & h_{1} \ldots h_{s}\left\{\eta_{0}+\sum_{j=1}^{s} \frac{d h_{j}}{h_{j}} \wedge \eta_{j}+\ldots\right. \\
& \left.\cdots+\sum \frac{d h_{j_{1}}}{h_{j_{1}}} \wedge \cdots \wedge \frac{d h_{j_{k}}}{h_{j_{k}}} \wedge \eta_{j_{1}, \ldots, j_{k}}\right\},
\end{aligned}
$$

where $\eta_{0} \in \Omega_{\mathbb{F}_{q}[x] / \mathbb{F}_{q}}^{k}$ and $\eta_{j} \in \Omega_{\mathbb{F}_{q}[x] / \mathbb{F}_{q}}^{k-1}$ and $\eta_{j_{1}, \ldots, j_{t}} \in \Omega_{\mathbb{F}_{q}[x] / \mathbb{F}_{q}}^{k-t}$ for $1 \leq j_{1}<\cdots<$ $j_{t} \leq s$ and $t=2, \ldots, k$.

Proof. See [12], Lemma 2.2.3.

The main result of this section is a generalization of Proposition 3.6, which will be used in the computation of the cohomology of the double complex.

Proposition 3.7. Let $h_{1}, \ldots, h_{s}$ be as above. Fix an integer $S$ so that $0 \leq S \leq s$ and suppose we have $\eta \in \Omega_{\mathbb{F}_{q}[x] / \mathbb{F}_{q}}^{k}$, for $0 \leq k \leq n-2-S$, such that

$$
d h_{j} \wedge d h_{1} \wedge \cdots \wedge d h_{S} \wedge \eta \equiv 0 \quad\left(\bmod h_{j}, h_{1}, \ldots, h_{S}\right)
$$

for $S+1 \leq j \leq s$. Then $\eta$ is of the form

$$
\begin{aligned}
\eta & =\sum_{i=1}^{S} h_{i} \gamma_{i}+\sum_{i=1}^{S} d h_{i} \wedge \xi_{i} \\
& +h_{S+1} \ldots h_{s}\left\{\eta_{0}+\sum_{j=S+1}^{s} \frac{d h_{j}}{h_{j}} \wedge \eta_{j}+\sum_{S+1 \leq j_{1}<j_{2} \leq s} \frac{d h_{j_{1}}}{h_{j_{1}}} \wedge \frac{d h_{j_{2}}}{h_{j_{2}}} \wedge \eta_{j_{1}, j_{2}}+\ldots\right\},
\end{aligned}
$$

where $\gamma_{i} \in \Omega_{\mathbb{F}_{q}[x] / \mathbb{F}_{q}}^{k}, \xi_{i} \in \Omega_{\mathbb{F}_{q}[x] / \mathbb{F}_{q}}^{k-1}$, and $\eta_{j_{1}, \ldots, j_{t}} \in \Omega_{\mathbb{F}_{q}[x] / \mathbb{F}_{q}}^{k-t}$ for $0 \leq t \leq k$.

Proof. The proof proceeds by an induction on $S$. As a basis step the case $S=0$ is Proposition 3.6. Suppose now that the proposition is true for some $S>0$ and also suppose that

$$
d h_{j} \wedge d h_{1} \wedge \cdots \wedge d h_{S} \wedge d h_{S+1} \wedge \eta \equiv 0 \quad\left(\bmod \left(h_{j}, h_{1}, \ldots, h_{S+1}\right)\right)
$$

for $S+2 \leq j \leq s$. To show that the proposition is true for $S+1$ we use induction on $s-(S+1)$, the number of factors in $h_{S+2} \ldots h_{s}$. For the basis step let $s=S+2$, 
so that $d h_{1} \wedge \cdots \wedge d h_{S+1} \wedge d h_{S+2} \wedge \eta \equiv 0\left(\bmod \left(h_{1}, \ldots, h_{S+2}\right)\right)$. By Proposition 3.5 we can write

$$
\eta=\sum_{i=1}^{S+1} h_{i} \gamma_{i}^{\prime}+\sum_{i=1}^{S+1} d h_{i} \wedge \xi_{i}^{\prime}+h_{S+2} \gamma_{S+2}^{\prime}+d h_{S+2} \wedge \xi_{S+2}^{\prime}
$$

for some $\gamma_{i}^{\prime}, \xi_{i}^{\prime}$. Therefore

$$
\eta=\sum_{i=1}^{S+1} h_{i} \gamma_{i}^{\prime}+\sum_{i=1}^{S+1} d h_{i} \wedge \xi_{i}^{\prime}+h_{S+2}\left\{\gamma_{S+2}^{\prime}+\frac{d h_{S+2}}{h_{S+2}} \wedge \xi_{S+2}^{\prime}\right\}
$$

which proves the proposition for $s-(S+1)=1$ and verifies the basis step of the induction (on $s-(S+1)$ ).

For the induction step, we assume that the proposition is true for $S+1$ and $s-1$. In this case we may write

$$
\eta=\sum_{i=1}^{S+1} h_{i} \gamma_{i}+\sum_{i=1}^{S+1} d h_{i} \wedge \xi_{i}+h_{S+2} \ldots h_{s-1}\left\{\eta_{0}+\sum_{S+2 \leq i \leq s-1} \frac{d h_{i}}{h_{i}} \wedge \eta_{i}+\ldots\right\} .
$$

We also assume that

$$
d h_{j} \wedge d h_{1} \wedge \cdots \wedge d h_{S+1} \wedge \eta \equiv 0 \quad\left(\bmod \left(h_{j}, h_{1}, \ldots, h_{S+1}\right)\right)
$$

for $j=S+2, \ldots, s$. We want to show that the proposition is now true for $s$. In order to do this we let $N$ be the largest integer such that $\eta_{i_{1} \ldots i_{t}}=0$ for any $t \geq N+1$ in (3-11) and proceed by induction on $N$.

Suppose $N=0$. Then we can write (3-11) as

$$
\eta=\sum_{i=1}^{S+1} h_{i} \gamma_{i}+\sum_{i=1}^{S+1} d h_{i} \wedge \xi_{i}+\left(h_{S+2} \ldots h_{s-1}\right) \eta_{0}
$$

By assumption $d h_{s} \wedge d h_{1} \wedge \cdots \wedge d h_{S+1} \wedge \eta \equiv 0\left(\bmod \left(h_{s}, h_{1}, \ldots, h_{S+1}\right)\right)$, which implies $\left(h_{S+2} \ldots h_{s-1}\right) d h_{s} \wedge d h_{1} \wedge \ldots \wedge d h_{S+1} \wedge \eta_{0} \equiv 0\left(\bmod \left(h_{s}, h_{1}, \ldots, h_{S+1}\right)\right)$. Since we are trying to verify the proposition for $S+1$, we are using the hypothesis $k \leq n-2-(S+1)=n-(S+3)$. Therefore $S+3 \leq n-k$.

The normal crossing divisor hypothesis assures that any sequence of $h_{i}$ 's of length less than $n$ is a regular sequence, so in particular any sequence of length $S+3$ will be a regular sequence. It follows that the sequence $\left(h_{S+2}, h_{s}, h_{1}, \ldots, h_{S+1}\right)$ is regular and therefore in the above relation we can make the substitution $h_{S+2} Q=h_{s} Q_{s}+$ $h_{1} Q_{1}+\cdots+h_{S+1} Q_{S+1}$, for some $Q_{i}$ 's, where $Q$ is in the ideal $\left(h_{s}, h_{1}, \ldots, h_{S+1}\right)$. In this case we can then remove the factor of $h_{S+2}$. We can repeat the argument with $h_{S+3}$ in place of $h_{S+2}$, then $h_{S+4}$ and so on, up to $h_{s-1}$. Therefore we can remove the factor $h_{S+2} \ldots h_{s-1}$, and the above relation reduces to $d h_{s} \wedge d h_{1} \wedge \ldots \wedge$ $d h_{S+1} \wedge \eta_{0} \equiv 0\left(\bmod \left(h_{s}, h_{1}, \ldots, h_{S+1}\right)\right)$. Now by applying Proposition 3.5 , it will follow that $\eta_{0}$ is congruent to 0 in the ideal $\left(h_{s}, h_{1}, \ldots, h_{S+1}, d h_{s}, d h_{1}, \ldots, d h_{S+1}\right)$, so that $\eta_{0}=\sum_{i=1}^{S+1} h_{i} \gamma_{i}^{\prime}+\sum_{i=1}^{S+1} d h_{i} \wedge \xi_{i}^{\prime}+h_{s} \gamma_{s}^{\prime}+d h_{s} \wedge \xi_{s}^{\prime}$ for some $\gamma_{1}^{\prime}, \ldots, \gamma_{s}^{\prime}$ and $\xi_{1}^{\prime}, \ldots, \xi_{s}^{\prime}$. 
Substituting into (3-12) we get

$$
\begin{aligned}
\eta & =\sum_{j=1}^{S+1} h_{j} \gamma_{j}+\sum_{j=1}^{S+1} d h_{j} \wedge \xi_{j} \\
& +h_{S+2} \ldots h_{s-1}\left\{\sum_{j=1}^{S+1} h_{j} \gamma_{j}^{\prime}+\sum_{j=1}^{S+1} d h_{j} \wedge \xi_{j}^{\prime}+h_{s} \gamma_{s}^{\prime}+d h_{s} \wedge \xi_{s}^{\prime}\right\} .
\end{aligned}
$$

Therefore $\eta$ is of the form

$$
\eta=\sum_{j=1}^{S+1} h_{j} \gamma_{j}^{\prime \prime}+\sum_{j=1}^{S+1} d h_{j} \wedge \xi_{j}^{\prime \prime}+h_{S+2} \ldots h_{s-1} h_{s}\left\{\gamma_{s}^{\prime}+\frac{d h_{s}}{h_{s}} \wedge \xi_{s}^{\prime}\right\}
$$

where $\gamma_{j}^{\prime \prime}=\gamma_{j}+\left(h_{S+2} \ldots h_{s-1}\right) \gamma_{j}^{\prime}$ and $\xi_{j}^{\prime \prime}=\xi_{j}+\left(h_{S+2} \ldots h_{s-1}\right) \xi_{j}^{\prime}$, which proves the proposition for $N=0$.

For the induction step we assume that the proposition is true for $N-1$ (i.e. true for $S+1, s-1$ and $N-1$ ). Suppose $\eta$ is in the form

$$
\begin{aligned}
\eta=\sum_{j=1}^{S+1} h_{j} \gamma_{j} & +\sum_{j=1}^{S+1} d h_{j} \wedge \xi_{j}+h_{S+2} \ldots h_{s-1}\left\{\eta_{0}+\sum_{j=S+2}^{s-1} \frac{d h_{j}}{h_{j}} \wedge \eta_{j}+\ldots\right. \\
& \left.+\sum_{S+2 \leq j_{1}<\cdots<j_{N} \leq s-1} \frac{d h_{j_{1}}}{h_{j_{1}}} \wedge \cdots \wedge \frac{d h_{j_{N}}}{h_{j_{N}}} \wedge \eta_{j_{1} \ldots j_{N}}\right\}
\end{aligned}
$$

and also suppose for $S+2 \leq j \leq s$ that

$$
d h_{j} \wedge d h_{1} \wedge \cdots \wedge d h_{S+1} \wedge \eta \equiv 0 \quad\left(\bmod \left(h_{j}, h_{1}, \ldots, h_{S+1}\right)\right) .
$$

For simplicity we consider the index $\left(j_{1}, \ldots, j_{N}\right)=(S+2, \ldots, S+N+1)$ in the last term of (3-13). In this case,

$$
\begin{aligned}
& \eta-\left(h_{S+N+2} \ldots h_{s-1}\right) d h_{S+2} \wedge \cdots \wedge d h_{S+N+1} \wedge \eta_{S+2, \ldots, S+N+1} \\
& \equiv \sum_{i=1}^{S+1} h_{i} \gamma_{i}+\sum_{i=1}^{S+1} d h_{i} \wedge \xi_{i} \quad\left(\bmod \left(h_{S+2}, \ldots, h_{S+N+1}\right)\right) .
\end{aligned}
$$

Multiplying both sides by $d h_{s} \wedge d h_{1} \wedge \cdots \wedge d h_{S+1}$ it follows that

$$
\begin{gathered}
\left(h_{S+N+2} \ldots h_{s-1}\right) d h_{s} \wedge d h_{1} \wedge \ldots \wedge d h_{S+N+1} \wedge \eta_{S+2, \ldots, S+N+1} \\
\equiv 0 \quad\left(\bmod \left(h_{s}, h_{1}, \ldots, h_{S+N+1}\right)\right) .
\end{gathered}
$$

We can remove the factor $h_{S+N+2} \ldots h_{s-1}$ using the same method as above.

Now, it follows by Proposition 3.5 that there exist $\widetilde{\gamma}_{i}$ and $\widetilde{\xi}_{i}$ for $i=1, \ldots, S+N+1$ and $i=s$, such that

$$
\eta_{S+2, \ldots, S+N+1}=\sum_{i=1}^{S+N+1} h_{i} \widetilde{\gamma}_{i}+\sum_{i=1}^{S+N+1} d h_{i} \wedge \widetilde{\xi}_{i}+h_{s} \widetilde{\gamma}_{s}+d h_{s} \wedge \widetilde{\xi}_{s} .
$$


Similarly for any index $\left(j_{1}, \ldots, j_{N}\right)$ of length $N$ we can write

$$
\begin{aligned}
\eta_{j_{1}, \ldots, j_{N}} & =\sum_{k=1}^{S+1} h_{k} \widetilde{\gamma}_{j_{1}, \ldots, j_{N} ; k}+\sum_{k=1}^{S+1} d h_{k} \wedge \widetilde{\xi}_{j_{1}, \ldots, j_{N} ; k} \\
& +\sum_{k=1}^{N} h_{j_{k}} \widehat{\gamma}_{j_{1}, \ldots, j_{N} ; k}+\sum_{k=1}^{N} d h_{j_{k}} \wedge \widehat{\xi}_{j_{1}, \ldots, j_{N} ; k} \\
& +h_{s} \widetilde{\gamma}_{j_{1}, \ldots, j_{N} ; s}+d h_{s} \wedge \widetilde{\xi}_{j_{1}, \ldots, j_{N} ; s} .
\end{aligned}
$$

Substitution into (3-13) yields

$$
\begin{aligned}
\eta=\sum_{j=1}^{S+1} h_{j} \gamma_{j} & +\sum_{j=1}^{S+1} d h_{j} \wedge \xi_{j}+h_{S+2} \ldots h_{s-1}\left\{\eta_{0}+\sum_{j=S+2}^{s-1} \frac{d h_{j}}{h_{j}} \wedge \eta_{j}+\ldots\right. \\
& +\sum_{S+2 \leq j_{1}<\cdots<j_{N-1} \leq s-1} \frac{d h_{j_{1}}}{h_{j_{1}}} \ldots \frac{d h_{j_{N-1}}}{h_{j_{N-1}}} \wedge \eta_{j_{1}, \ldots, j_{N-1}} \\
& +\sum_{S+2 \leq j_{1}<\cdots<j_{N} \leq s-1} \frac{d h_{j_{1}}}{h_{j_{1}}} \ldots \frac{d h_{j_{N}}}{h_{j_{N}}} \wedge\left\{\sum_{k=1}^{N} h_{j_{k}} \widetilde{\gamma}_{j_{1}, \ldots, j_{N} ; k}\right. \\
& \left.\left.+\sum_{k=1}^{N} d h_{j_{k}} \wedge \widetilde{\xi}_{j_{1}, \ldots, j_{N} ; k}+h_{s} \widetilde{\gamma}_{j_{1}, \ldots, j_{N} ; s}+d h_{s} \wedge \widetilde{\xi}_{j_{1}, \ldots, j_{N} ; s}\right\}\right\}
\end{aligned}
$$

Letting

$$
\begin{aligned}
\rho=\eta-h_{S+2} \ldots h_{s-1} & \left\{\sum _ { S + 2 \leq j _ { 1 } < \cdots < j _ { N } \leq s - 1 } \frac { d h _ { j _ { 1 } } } { h _ { j _ { 1 } } } \ldots \frac { d h _ { j _ { N } } } { h _ { j _ { N } } } \left(h_{s} \widetilde{\gamma}_{j_{1}, \ldots, j_{N} ; s}\right.\right. \\
& \left.\left.+d h_{s} \wedge \widetilde{\xi}_{j_{1}, \ldots, j_{N} ; s}\right)\right\}
\end{aligned}
$$

we see that $\rho$ satisfies the induction hypothesis and can therefore be written as

$$
\begin{aligned}
\rho & =\sum_{j=1}^{S+1} h_{j} \widehat{\gamma}_{j}+\sum_{j=1}^{S+1} d h_{j} \wedge \widehat{\xi}_{j} \\
& +h_{S+2} \ldots h_{s}\left\{\rho_{0}+\cdots+\sum_{S+2 \leq j_{1}<\cdots<j_{N-1} \leq s-1} \frac{d h_{j_{1}}}{h_{j_{1}}} \ldots \frac{d h_{j_{N-1}}}{h_{j_{N-1}}} \wedge \rho_{j_{1}, \ldots, j_{N-1}}\right\}
\end{aligned}
$$

for some $\widehat{\gamma}_{j}, \widehat{\xi}_{j}$ and $\rho_{0}, \ldots, \rho_{j_{1}, \ldots, j_{N-1}}$. Substituting this back into (3-16) it follows that $\eta$ is of the form

$$
\begin{aligned}
\eta & =\sum_{j=1}^{S+1} h_{j} \bar{\gamma}_{j}+\sum_{j=1}^{S+1} d h_{j} \wedge \bar{\xi}_{j} \\
& +h_{S+2} \ldots h_{s}\left\{\bar{\eta}_{0}+\cdots+\sum_{S+2 \leq j_{1}<\cdots<j_{N+1} \leq s} \frac{d h_{j_{1}}}{h_{j_{1}}} \ldots \frac{d h_{j_{N+1}}}{h_{j_{N+1}}} \wedge \bar{\eta}_{j_{1}, \ldots, j_{N+1}}\right\}
\end{aligned}
$$


for some $\bar{\gamma}_{j}, \bar{\xi}_{j}, \bar{\eta}_{j}, \ldots, \bar{\eta}_{j_{1}, \ldots, j_{N+1}}$ which closes the triple induction and proves the proposition.

3.3. Computation of horizontal cohomology. From the results of Section 3.1, we have that the cohomology of the double complex (3-8) is equal to the cohomology of the complex across the top row (after the vertical cohomology has been computed),

$$
0 \longrightarrow H^{r}\left(\left(\boldsymbol{\Omega}^{(0, \bullet)}\right)^{(D)}, \partial_{v}\right) \stackrel{\partial_{h}}{\longrightarrow} \ldots \stackrel{\partial_{h}}{\longrightarrow} H^{r}\left(\left(\boldsymbol{\Omega}^{(n, \bullet)}\right)^{(D)}, \partial_{v}\right) \longrightarrow 0,
$$

where $H^{k}\left(\Omega_{\mathbb{F}_{q}[x, y] / \mathbb{F}_{q}}\langle Y\rangle^{(0)}, d_{\langle Y\rangle} F \wedge\right) \cong H^{k-r}\left(H^{r}\left(\left(\mathbf{\Omega}^{(\bullet, \bullet)}\right)^{(D)}, \partial_{v}\right), \partial_{h}\right)$.

Along with (3-1) and (3-2), let $\operatorname{deg}_{2}$ be the second half of a bigrading $\left(\operatorname{deg}_{1}, \operatorname{deg}_{2}\right)$ on $\Omega_{\mathbb{F}_{q}[x, y] / \mathbb{F}_{q}}^{k}\langle Y\rangle$ by setting

$$
\begin{gathered}
\operatorname{deg}_{2} x_{i}=\operatorname{deg}_{2} d x_{i}=0, \quad \text { for } i=1, \ldots, n \quad \text { and } \\
\operatorname{deg}_{2} y_{j}=\operatorname{deg}_{2} d y_{j}=1, \quad \text { for } j=1, \ldots, r .
\end{gathered}
$$

From (3-8) and the definition of $\partial_{v}$, we have in the top position of each column (separating out powers of the $y_{j}$ 's)

$$
H^{r}\left(\left(\boldsymbol{\Omega}^{(\ell, \bullet)}\right)^{(D)}, \partial_{v}\right)
$$

$$
=\bigoplus_{\left\{1 \leq i_{1}<\cdots<i_{\ell} \leq n\right\}} \bigoplus_{\left\{v_{j} \geq 0\right\}} y_{1}^{v_{1}} \ldots y_{r}^{v_{r}}\left[\mathbb{F}_{q}[x] /(I)\right]^{\left(D-\ell+\sum v_{j} d_{j}\right)} d x_{i_{1}} \ldots d x_{i_{\ell}} \frac{d y_{1}}{y_{1}} \ldots \frac{d y_{r}}{y_{r}}
$$

where $I$ is the ideal of $\mathbb{F}_{q}[x]$ generated by the $f_{j}$ for which $v_{j}>0$.

Since $\partial_{h}$ shifts the bidegree by $(0,1)$, we compute the cohomology of sequences of the form

$$
0 \longrightarrow H^{r}\left(\boldsymbol{\Omega}^{(0, \bullet)}\right)^{(D, J)} \stackrel{\partial_{h}}{\longrightarrow} \ldots \stackrel{\partial_{h}}{\longrightarrow} H^{r}\left(\boldsymbol{\Omega}^{(n, \bullet)}\right)^{(D, J+n)} \longrightarrow 0,
$$

where

$$
H^{r}\left(\left(\boldsymbol{\Omega}^{(\ell, \bullet)}\right)^{(D)}\right)=\bigoplus_{J=0}^{\infty} H^{r}\left(\left(\boldsymbol{\Omega}^{(\ell, \bullet)}\right)^{(D, J)}\right) .
$$

We can now state the main result of this section.

Theorem 3.2. If $f_{1}, \ldots, f_{r} \in \mathbb{F}_{q}\left[x_{1}, \ldots, x_{n}\right]$ satisfy the definition of a divisor with normal crossings in $\mathbb{P}^{n-1}\left(\mathbb{F}_{q}\right)$, then

$$
H^{\ell}\left(H^{r}\left(\mathbf{\Omega}^{(\bullet, \bullet)}\right)^{(D)}, \partial_{h}\right)=0 \quad \text { for } \ell<n-1 .
$$

Proof. We break up the proof into cases based on the value of $\ell$ and the value of $J$.

Case $1 . J=0$ and $0 \leq \ell \leq n-2$. Consider the sequence

$$
H^{r}\left(\left(\boldsymbol{\Omega}^{(\ell-1, \bullet)}\right)^{(D,-1)}\right) \stackrel{\partial_{h}}{\longrightarrow} H^{r}\left(\left(\boldsymbol{\Omega}^{(\ell, \bullet)}\right)^{(D, 0)}\right) \stackrel{\partial_{h}}{\longrightarrow} H^{r}\left(\left(\boldsymbol{\Omega}^{(\ell+1, \bullet)}\right)^{(D, 1)}\right) .
$$


It is clear that the negative value in $\operatorname{deg}_{2}$ implies that the left most space is zero, so we compute the kernel of the map

$$
\begin{aligned}
\partial_{h}: & \bigoplus_{\left\{1 \leq i_{1}<\cdots<i_{\ell} \leq n\right\}} \mathbb{F}_{q}[x]^{(D-\ell)} d x_{i_{1}} \ldots d x_{i_{\ell}} \frac{d y_{1}}{y_{1}} \ldots \frac{d y_{r}}{y_{r}} \\
& \longrightarrow \bigoplus \bigoplus_{j=1}^{r} y_{j}\left[\mathbb{F}_{q}[x] /\left(f_{j}\right)\right]^{\left(D-\ell+d_{j}-1\right)} d x_{i} d x_{i_{1}} \ldots d x_{i_{\ell}} \frac{d y_{1}}{y_{1}} \ldots \frac{d y_{r}}{y_{r}}
\end{aligned}
$$

where the outer sum of the bottom row is over all subsets of $\{1, \ldots, n\}$ of the type $i \cup\left\{i_{1}, \ldots, i_{\ell}\right\}$.

Let $\omega \in \bigoplus \mathbb{F}_{q}[x]^{(D-\ell)} d x_{i_{1}} \ldots d x_{i_{\ell}} \frac{d y_{1}}{y_{1}} \ldots \frac{d y_{r}}{y_{r}}$, where the sum is over all indices $1 \leq i_{1}<\cdots<i_{\ell} \leq n$. Now $\omega$ is in the kernel of $\partial_{h}$ if and only if $d f_{j} \wedge \omega \equiv 0$ $\left(\bmod f_{j}\right)$ for every $j$. Since we are assuming that $\ell \leq n-2$, we can apply Proposition 3.6 and we can write $\omega$ as (dropping the $d y / y$ 's)

$$
\omega=\left(f_{1} \ldots f_{r}\right)\left\{\omega_{0}+\sum_{1}^{r} \frac{d f_{j}}{f_{j}} \wedge \omega_{j}+\sum \frac{d f_{j_{1}}}{f_{j_{1}}} \wedge \frac{d f_{j_{2}}}{f_{j_{2}}} \wedge \omega_{j_{1}, j_{2}}+\ldots\right\},
$$

where $\omega_{0}$ is an $\ell$-form, each of the $\omega_{j}$ 's is an $(\ell-1)$-form and each $\omega_{j_{1}, j_{2}}$ is an $(\ell-2)$-form, and so on. Moreover, these are the only such $\omega$.

The first summand $\left(f_{1} \ldots f_{r}\right) \omega_{0}$ has a value of $\operatorname{deg}_{1}$ that is too large, as $\operatorname{deg}_{1}=$ $\sum d_{j}>D$, so that $\omega_{0}=0$. In the subsequent terms $\left(f_{1} \ldots f_{r}\right) \sum_{j=1}^{r} \frac{d f_{j}}{f_{j}} \wedge \omega_{j}$, we have for any $j$,

$$
\operatorname{deg}_{1}\left(\left(f_{1} \ldots \hat{f}_{j} \ldots f_{r}\right) d f_{j} \wedge \omega_{j}\right) \geq \sum_{j=1}^{r} d_{j}>D
$$

since $\operatorname{deg}_{1} f_{j}=\operatorname{deg}_{1} d f_{j}$.

Similarly, in each of the remaining terms we have

$$
\operatorname{deg}_{1}\left(\left(f_{1} \ldots f_{r}\right) \frac{d f_{j_{1}}}{f_{j_{1}}} \ldots \frac{d f_{j_{t}}}{f_{j_{t}}}\right)=d_{1}+\cdots+d_{r}
$$

Now since $D<d_{1}+\cdots+d_{r}$ we have that

$$
\operatorname{deg}_{1}\left(\left(f_{1} \ldots f_{r}\right) \frac{d f_{j_{1}}}{f_{j_{1}}} \ldots \frac{d f_{j_{t}}}{f_{j_{t}}}\right)>D
$$

which is a contradiction of the choice of coefficients in $\mathbb{F}_{q}[x]^{(D-\ell)}$. By this contradiction, if any such form is in the kernel of $\partial_{h}$ it is necessarily zero. Therefore $H^{k}\left(H^{r}\left(\left(\mathbf{\Omega}^{(\bullet, \bullet)}\right)^{(D, 0)}, \partial_{h}\right)\right)=0$ for $k<n-1$.

Case 2. $1 \leq J$ and $0 \leq \ell \leq n-2$. We are considering forms with one or more factors of the $y_{j}$ raised to varying powers $v_{j}$. As before $J=\sum_{j=1}^{r} v_{j}$. We let $\kappa$ be an integer, $1 \leq \kappa \leq r$, that represents the number of factors of $y_{j}$ in a given form with non-zero exponents. For each of these products as $y_{j_{1}}^{v_{j_{1}}} \ldots y_{j_{\kappa}}^{v_{j_{\kappa}}}$ we associate the form $\omega\left(0, \ldots, v_{j_{1}}, \ldots, 0, v_{j_{\kappa}}, \ldots, 0\right)$. We will condense the $r$-tuple $\left(0, \ldots, v_{j_{1}}, \ldots, 0, v_{j_{\kappa}}, \ldots, 0\right)$ to the $\kappa$-tuple of non-zero terms $\left(v_{j_{1}}, \ldots, v_{j_{\kappa}}\right)$ when the context allows and write $\omega\left(v_{j_{1}}, \ldots, v_{j_{\kappa}}\right)$. 
For fixed $J$, we index the terms of $\omega \in H^{r}\left(\left(\boldsymbol{\Omega}^{(\ell, \bullet)}\right)^{(D, J)}\right)$ by ascending order of $\kappa$ and write

$$
\begin{aligned}
\omega= & \sum_{j=1}^{r} y_{j}^{J} \omega(0, \ldots, J, \ldots, 0)+\sum_{\left\{1 \leq j_{1}<j_{2} \leq r\right\}} \sum_{\left\{v_{j_{1}}+v_{j_{2}}=J\right\}} y_{j_{1}}^{v_{j_{1}}} y_{j_{2}}^{v_{j_{2}}} \omega\left(v_{j_{1}}, v_{j_{2}}\right) \\
& +\cdots+\sum_{1 \leq j_{1}<\cdots<j_{J} \leq r} y_{j_{1}} \ldots y_{j_{J}} \omega(0, \ldots, 1, \ldots, 0, \ldots 1) .
\end{aligned}
$$

The forms in (3-25) will satisfy (again dropping the factor of $\frac{d y_{1}}{y_{1}} \ldots \frac{d y_{r}}{y_{r}}$ )

$$
\omega\left(v_{j_{1}}, \ldots, v_{j_{\kappa}}\right) \in \bigoplus_{1 \leq i_{1}<\cdots<i_{\ell} \leq n}\left[\mathbb{F}_{q}[x] /\left(f_{j_{1}}, \ldots, f_{j_{\kappa}}\right)\right]^{\left(D+v_{j_{1}} d_{j_{1}}+\cdots+v_{j_{\kappa}} d_{j_{\kappa}}-\ell\right)} d x_{i_{1}} \ldots d x_{i_{\ell}} .
$$

Suppose now that for a differential form $\omega$ as in (3-25) we have $\omega \in \operatorname{ker}\left(\partial_{h}\right)$. Consider first the summand of $\omega$ where $\kappa=1$. If we fix one of the indices $j$ such that $\omega(J)=\omega(0, \ldots, J, \ldots, 0) \neq 0$ and apply $\partial_{h}$ we get $\left(y_{1} d f_{1}+\cdots+y_{r} d f_{r}\right) \wedge y_{j}^{J} \omega(J)=$ $y_{1} y_{j}^{J} d f_{1} \wedge \omega(J)+\cdots+y_{j}^{J+1} d f_{j} \wedge \omega(J)+\cdots+y_{r} y_{j}^{J} d f_{r} \wedge \omega(J)$. As in the first case, for each $j=1, \ldots, r$, the resulting $(\ell+1)$-form associated to the factor $y_{j}^{J+1}$ satisfies $d f_{j} \wedge \omega(J) \equiv 0\left(\bmod f_{j}\right)$. Hence, by Proposition 3.6 , there exists $\eta(J) \in \boldsymbol{\Omega}_{\mathbb{F}_{q}[x] / \mathbb{F}_{q}}^{(\ell-1)}$ such that $\omega(J) \equiv d f_{j} \wedge \eta(J)\left(\bmod f_{j}\right)$. Making this substitution for each $j=1, \ldots, r$, we conclude that summands of the type (the first sum on the right-hand side of $(3-25))$ are in the image of $\partial_{h}$ and that $\omega$ can be reduced to the form $\omega=$

$\sum_{\left\{1 \leq j_{1}<j_{2} \leq r\right\}} \sum_{\left\{v_{j_{1}}+v_{j_{2}}=J\right\}} y_{j_{1}}^{v_{j_{1}}} y_{j_{2}}^{v_{j_{2}}} \omega\left(v_{j_{1}}, v_{j_{2}}\right)+\cdots+\sum_{1 \leq j_{1}<\cdots<j_{J} \leq r} y_{t_{1}} \ldots y_{t_{J}} \omega(1, \ldots, 1)$.

Now for $2 \leq \kappa<J$, any monomial in $\sum y_{j} d f_{j} \wedge \omega$ that contains only $y_{j_{1}}, \ldots, y_{j_{\kappa}}$ comes from sums of forms of the type $y_{j_{t}} d f_{j_{t}} \wedge y_{j_{1}}^{v_{j_{1}}} \ldots y_{j_{\kappa}}^{v_{j_{\kappa}}} \omega\left(v_{j_{1}}, \ldots, v_{j_{\kappa}}\right)$, involving some or all of the $y_{j_{1}}, \ldots, y_{j_{\kappa}}$. Since we are assuming $\omega \in \operatorname{ker} \partial_{h}$, we will have

$$
\sum_{t=1}^{\kappa} y_{j_{t}} d f_{j_{t}} \wedge \sum_{|v|=J, v_{j_{t}}>0} y_{j_{1}}^{v_{j_{1}}} \ldots y_{j_{\kappa}}^{v_{j_{\kappa}}} \omega\left(v_{j_{1}}, \ldots, v_{j_{\kappa}}\right) \equiv 0 \quad\left(\bmod \left(f_{j_{1}}, \ldots, f_{j_{\kappa}}\right)\right) .
$$

If $J \leq n-2$ we can apply Proposition 3.4 to find forms $\eta\left(v_{j_{1}}^{\prime}, \ldots, v_{j_{\kappa}}^{\prime}\right)$ such that

$$
\begin{gathered}
\sum_{|v|=J, v_{j_{t}}>0} y_{j_{1}}^{v_{j_{1}}-1} \ldots y_{j_{\kappa}}^{v_{j_{\kappa}}-1} \omega\left(v_{j_{1}}, \ldots, v_{j_{\kappa}}\right) \\
\equiv \sum_{t=1}^{\kappa} y_{j_{t}} d f_{t_{j}} \wedge \sum_{\left|v^{\prime}\right|=J-\kappa-1} y_{j_{1}}^{v_{j_{1}}^{\prime}} \ldots y_{j_{\kappa}}^{v_{j_{\kappa}}^{\prime}} \eta\left(v_{j_{1}}^{\prime}, \ldots, v_{j_{\kappa}}^{\prime}\right) \quad\left(\bmod \left(f_{t_{1}}, \ldots, f_{t_{\kappa}}\right)\right),
\end{gathered}
$$

where the sum is over the set of all monomials containing only $y_{j_{1}}, \ldots, y_{j_{\kappa}}$ having $\sum d_{j_{t}} v_{j_{t}}^{\prime}=J-1-\kappa$.

If $J \geq n-1$, the quotients $\left[\mathbb{F}_{q}[x] /\left(f_{j_{1}}, \ldots, f_{j_{\kappa}}\right)\right]^{\left(D+v_{j_{1}} d_{j_{1}}+\cdots+v_{j_{\kappa}} d_{j_{\kappa}}-\ell\right)}$ will all be zero. The degree of the Hilbert polynomial of the quotient is $\sum_{j=1}^{\kappa} d_{j}-\kappa$ which is less than $D+v_{j_{1}} d_{j_{1}}+\cdots+v_{j_{\kappa}} d_{j_{\kappa}}-\ell$ if $J \geq n-1,0 \leq \ell \leq n-2$ and $D>0$.

If we now let

$$
\eta=y_{j_{1}} \ldots y_{j_{\kappa}} \sum_{\left|v^{\prime}\right|=J-\kappa-1, v_{j_{t}}^{\prime} \geq 0} y_{j_{1}}^{v_{j_{1}}^{\prime}} \ldots y_{j_{\kappa}}^{v_{j_{\kappa}}^{\prime}} \eta\left(v_{j_{1}}^{\prime}, \ldots, v_{j_{\kappa}}^{\prime}\right),
$$


then $\omega-\sum_{j=1}^{r} y_{j} d f_{j} \wedge \eta$ has no terms involving exactly the variables $y_{j_{1}}, \ldots, y_{j_{\kappa}}$. This argument can be repeated for any terms in (3-25) involving fewer than $\kappa$ different $y_{j}$ 's, so by induction on $\kappa$, we can reduce to the case where

$$
\omega=\sum_{1 \leq j_{1}<\cdots<j_{J} \leq r} y_{j_{1}} \ldots y_{j_{J}} \omega\left(j_{1}, \ldots, j_{J}\right),
$$

where any remaining terms contain $J$ distinct $y_{j}$ 's all of exponent 1 .

Here we will no longer index by the exponent $v_{j}$, since they are either 0 or 1 , but rather by the subscript $j$ for which there is a factor of $y_{j}$.

We will reduce $\omega$ further by using induction on the number of terms of the form $y_{j_{1}} \ldots y_{j_{J}} \omega\left(j_{1}, \ldots, j_{J}\right)$. Suppose that $\omega$ has one term, say $\omega=y_{1} \ldots y_{J} \omega(1, \ldots, J)$. Then

$$
\begin{aligned}
\sum_{j=1}^{r} y_{j} d f_{j} \wedge \omega & =\sum_{j=1}^{J} y_{1} \ldots y_{j}^{2} \ldots y_{J} d f_{j} \wedge \omega(1, \ldots, J) \\
& +\sum_{j=J+1}^{r} y_{j} y_{1} \ldots y_{J} d f_{j} \wedge \omega(1, \ldots, J)
\end{aligned}
$$

If $\omega \in \operatorname{ker}\left(\partial_{h}\right)$ we can deduce from the top sum on the right in (3-26) that $d f_{j} \wedge$ $\omega(1, \ldots, J) \equiv 0\left(\bmod \left(f_{1}, \ldots, f_{J}\right)\right)$ and therefore by Proposition 3.3 there exists $\eta \in \Omega_{\mathbb{F}_{q}[x] / \mathbb{F}_{q}}^{\ell-J}$ such that

$$
\omega(1, \ldots, J) \equiv d f_{1} \wedge \cdots \wedge d f_{J} \wedge \eta \quad\left(\bmod \left(f_{1}, \ldots, f_{J}\right)\right) .
$$

Now substituting this into the lower sum on the right in (3-26) we have

$$
\left(y_{j} y_{1} \ldots y_{J}\right) d f_{j} \wedge d f_{1} \wedge \cdots \wedge d f_{J} \wedge \eta \equiv 0 \quad\left(\bmod f_{j}, f_{1}, \ldots, f_{J}\right),
$$

which implies

$$
d f_{j} \wedge d f_{1} \wedge \cdots \wedge d f_{J} \wedge \eta \equiv 0 \quad\left(\bmod f_{j}, f_{1}, \ldots, f_{J}\right)
$$

for $J+1 \leq j \leq r$.

Using Proposition 3.7 we can write

$$
\begin{aligned}
\eta & =\sum_{j=1}^{J} f_{j} \gamma_{j}+\sum_{j=1}^{J} d f_{j} \wedge \xi_{j} \\
& +f_{J+1} \ldots f_{r}\left\{\eta_{0}+\sum_{j=J+1}^{r} \frac{d f_{j}}{f_{j}} \wedge \eta_{j}+\sum_{J+1 \leq j_{1}<j_{2} \leq r} \frac{d f_{j_{1}}}{f_{j_{1}}} \wedge \frac{d f_{j_{2}}}{f_{j_{2}}} \wedge \eta_{j_{1}, j_{2}}+\ldots\right\},
\end{aligned}
$$

where $\gamma_{j} \in \Omega_{\mathbb{F}_{q}[x] / \mathbb{F}_{q}}^{(\ell-J)}, \xi_{j} \in \Omega_{\mathbb{F}_{q}[x] / \mathbb{F}_{q}}^{(\ell-J-1)}$, and $\eta_{j_{1}, \ldots, j_{s}} \in \Omega_{\mathbb{F}_{q}[x] / \mathbb{F}_{q}}^{(\ell-J-s)}$.

If we let $\eta^{\prime}=\eta-\left[\sum_{j=1}^{J} f_{j} \gamma_{j}+\sum_{j=1}^{J} d f_{j} \wedge \xi_{j}\right]$, then (3-27) holds with $\eta$ replaced by $\eta^{\prime}$. Also $d f_{j} \wedge \eta^{\prime} \equiv 0\left(\bmod f_{j}\right)$, for each $j=J+1, \ldots, r$. Therefore $\partial_{h}\left(y_{2} \ldots y_{J} d f_{2} \wedge\right.$ $\left.\cdots \wedge d f_{J} \wedge \eta^{\prime}\right)=\omega$ so that $\omega$ is in the image of $\partial_{h}$.

For the induction step, we first put an ordering on the differential forms $y_{j_{1}}, \ldots, y_{j_{J}} \omega\left(j_{1}, \ldots, j_{J}\right)$. In this ordering we let $y_{1}^{v_{1}} \ldots y_{r}^{v_{r}}>y_{1}^{w_{1}} \ldots y_{r}^{w_{r}}$ if $v_{k}>w_{k}$ for some $k$ and $v_{j}=w_{j}$ for all $j<k$. Choose $t_{1}, \ldots, t_{J}$ so that the monomial $y_{t_{1}} \ldots y_{t_{J}}$ is maximal in the ordering among all of the remaining non-zero terms of $\omega$. 
Supposing that $\partial_{h}(\omega)=0$, it follows that $d f_{j} \wedge \omega\left(t_{1}, \ldots, t_{J}\right) \in\left(f_{t_{1}}, \ldots, f_{t_{J}}\right)$, for each $j \in\left\{t_{1}, \ldots, t_{J}\right\}$. On the other hand, for each $j \notin\left\{t_{1}, \ldots, t_{J}\right\}$, where $j<t_{1}$, we know that $d f_{j} \wedge \omega\left(t_{1}, \ldots, t_{J}\right)$ is the unique coefficient of $y_{j} y_{t_{1}} \ldots y_{t_{J}}$ in $\partial_{h}(\omega)$. If a term such as this should come from $d f_{t_{j}} \wedge \omega\left(j, t_{1}, \ldots, \hat{t}_{j} \ldots t_{J}\right)$ with $j<t_{1}$, then $y_{j} y_{t_{1}} \ldots \hat{y}_{t_{j}} \ldots y_{t_{J}}>y_{t_{1}} \ldots y_{t_{J}}$ in the ordering, which contradicts the maximality. By Proposition 3.3 we can write

$$
\omega\left(t_{1}, \ldots, t_{J}\right)=d f_{t_{1}} \wedge \cdots \wedge d f_{t_{J}} \wedge \eta\left(t_{1}, \ldots, t_{J}\right)
$$

for some $\eta\left(t_{1}, \ldots, t_{J}\right)$. Now by Proposition $3.7, \eta\left(t_{1}, \ldots, t_{J}\right)$ is of the form

$$
\begin{aligned}
\eta\left(t_{1}, \ldots, t_{J}\right) & =\sum_{i=1}^{J} f_{t_{i}} \gamma_{t_{i}}+\sum_{i=1}^{J} d f_{t_{i}} \wedge \xi_{t_{i}} \\
& +\left(f_{1} \ldots f_{t_{1}-1}\right)\left\{\eta_{0}+\sum_{i=1}^{t_{1}-1} \frac{d f_{i}}{f_{i}} \wedge \eta_{i}+\ldots\right\} .
\end{aligned}
$$

In the above, if we let $\tau\left(t_{1}, \ldots, t_{J}\right)=\left(f_{1} \ldots f_{t_{1}-1}\right)\left\{\eta_{0}+\sum_{i=1}^{t_{1}-1} \frac{d f_{i}}{f_{i}} \wedge \eta_{i}+\ldots\right\}$, then

$$
\begin{aligned}
\partial_{h}\left(y_{t_{2}} \ldots y_{t_{J}}\right. & \left(d f_{t_{2}} \wedge \cdots \wedge d f_{t_{J}} \wedge \tau\left(t_{1}, \ldots, t_{J}\right)\right) \\
& =\sum_{k<t_{1}} y_{k} y_{t_{2}} \ldots y_{t_{J}} d f_{k} \wedge d f_{t_{2}} \wedge \cdots \wedge d f_{t_{J}} \wedge \tau\left(t_{1}, \ldots, t_{J}\right) \\
& +y_{t_{1}} y_{t_{2}} \ldots y_{t_{J}} d f_{t_{1}} \wedge \cdots \wedge d f_{t_{J}} \wedge \tau\left(t_{1}, \ldots, t_{J}\right) \\
& +\sum_{k>t_{1}} y_{k} y_{t_{2}} \ldots y_{t_{J}} d f_{k} \wedge d f_{t_{2}} \wedge \cdots \wedge d f_{t_{J}} \wedge \tau\left(t_{1}, \ldots, t_{J}\right)
\end{aligned}
$$

so that $\omega-\partial_{h}\left(\sigma\left(t_{1}, \ldots, t_{J}\right)\right)$ will contain only monomials that are smaller in the ordering than the maximal $y_{t_{1}} \ldots y_{t_{J}}$. The terms in the first row on the righthand side of the equation are congruent to 0 modulo $\left(f_{k}, f_{t_{2}}, \ldots, f_{t_{J}}\right)$. The second row is $y_{t_{1}} \ldots y_{t_{J}} \omega\left(t_{1}, \ldots, t_{J}\right)$ and in the third row all of the terms are less than $y_{t_{1}} \ldots y_{t_{J}} \omega\left(t_{1}, \ldots, t_{J}\right)$ in the ordering. Therefore the maximal term is in the image of $\partial_{h}$ and won't contribute to the cohomology. This completes the induction step and finishes Case 2. Therefore $H^{k}\left(\left(H^{r}\left(\mathbf{\Omega}^{(\bullet, \bullet)}\right)\right)^{(D, J)}\right)=0$ for all $J$ and $k<n-$ 1 .

3.4. Dimensions $n+r-1$ and $n+r$. In this section we establish an isomorphism between the two remaining non-zero cohomology spaces.

Proposition 3.8. If $f_{1}, \ldots, f_{r}$ form a normal crossing divisor, then

$$
H^{n+r-1}\left(\Omega_{\mathbb{F}_{q}[x, y] / \mathbb{F}_{q}}\langle Y\rangle^{(D)}, d_{\langle Y\rangle} F \wedge\right) \cong H^{n+r}\left(\Omega_{\mathbb{F}_{q}[x, y] / \mathbb{F}_{q}}\langle Y\rangle^{(D)}, d_{\langle Y\rangle} F \wedge\right),
$$

and in particular, for each $J$,

$$
H^{n+r-1}\left(\Omega_{\mathbb{F}_{q}[x, y] / \mathbb{F}_{q}}\langle Y\rangle\right)^{(D, J)} \cong H^{n+r}\left(\Omega_{\mathbb{F}_{q}[x, y] / \mathbb{F}_{q}}\langle Y\rangle\right)^{(D, J)} .
$$

Moreover $H^{n+r}\left(\Omega_{\mathbb{F}_{q}[x, y] / \mathbb{F}_{q}}\langle Y\rangle\right)^{(D, J)}=0$ for all $J \geq n$.

Proof. Abbreviate the notation by letting $\Omega^{k}\langle Y\rangle^{(D)}=\Omega_{\mathbb{F}_{q}[x, y] / \mathbb{F}_{q}}^{k}\langle Y\rangle^{(D)}$. Following the method of Section 4 of [5] define the map $\Upsilon: \Omega^{k}\langle Y\rangle^{(D)} \longrightarrow \Omega^{k-1}\langle Y\rangle^{(D)}$ by 
linearity on the polynomials in $\mathbb{F}_{q}[x, y]^{(D-\ell)}$ and by the formula

$$
\begin{aligned}
& \Upsilon\left(d x_{i_{1}} \wedge \cdots \wedge d x_{i_{\ell}} \wedge \frac{d y_{j_{1}}}{y_{j_{1}}} \wedge \cdots \wedge \frac{d y_{j_{m}}}{y_{j_{m}}}\right) \\
& \left.\quad=\sum_{t=1}^{\ell}(-1)^{t-1} x_{i_{t}} d x_{i_{1}} \wedge \cdots \wedge \widehat{d x_{i_{t}}} \wedge \cdots \wedge d x_{i_{\ell}} \wedge \frac{d y_{j_{1}}}{y_{j_{1}}} \wedge \cdots \wedge \frac{d y_{j_{m}}}{y_{j_{m}}}\right) \\
& \quad+\sum_{s=1}^{m}(-1)^{\ell+s-1}\left(-d_{j_{s}}\right) d x_{i_{1}} \wedge \cdots \wedge d x_{i_{\ell}} \wedge \frac{d y_{j_{1}}}{y_{j_{1}}} \wedge \cdots \wedge \frac{\widehat{d y_{j_{s}}}}{y_{j_{s}}} \wedge \cdots \wedge \frac{d y_{j_{m}}}{y_{j_{m}}}
\end{aligned}
$$

where $\widehat{d x_{i}}$ and $\frac{\widehat{d y_{j_{s}}}}{y_{j_{s}}}$ are terms to be omitted.

Since $\Upsilon^{2}=0$ we can define the complex

$$
0 \longrightarrow \Omega^{n+r}\langle Y\rangle^{(D)} \stackrel{\Upsilon}{\longrightarrow} \ldots \stackrel{\Upsilon}{\longrightarrow} \Omega^{0}\langle Y\rangle^{(D)} \longrightarrow 0
$$

which is isomorphic to the Koszul complex on $\mathbb{F}_{q}[x, y]$ defined by the elements $x_{1}, \ldots x_{n},-d_{1}, \ldots,-d_{r}$.

It is a straightforward computation to show for any differential forms $\omega_{1}, \omega_{2}$, with $\omega_{1} \in \Omega^{k}\langle Y\rangle^{(D)}$, that $\Upsilon\left(\omega_{1} \wedge \omega_{2}\right)=\Upsilon\left(\omega_{1}\right) \wedge \omega_{2}+(-1)^{k} \omega_{1} \wedge \Upsilon\left(\omega_{2}\right)$. For a polynomial $f$ in $\mathbb{F}_{q}\left[x_{1}, \ldots, x_{n}\right]$ we have $\Upsilon\left(d_{\langle Y\rangle} f\right)=\operatorname{deg}(f) \cdot f$. Therefore if $F(x, y)=\sum_{j=1}^{r} y_{j} f_{j}(x)$ we have $\Upsilon\left(d_{\langle Y\rangle} F\right)=0$ and $\Upsilon\left(d_{\langle Y\rangle} F \wedge \omega\right)+d_{\langle Y\rangle} F \wedge \Upsilon(\omega)=$ 0 .

For $k=0, \ldots, n+r-1$ define the spaces $\widetilde{\Omega}^{k}\langle Y\rangle^{(D)}=\Upsilon\left(\Omega^{k+1}\langle Y\rangle^{(D)}\right)$. Since $x_{1}, \ldots, x_{n}$ form a regular sequence in $\mathbb{F}_{q}[x]$ of depth $n$ and since $\left(d_{j}, p\right)=1$ for each $j$, the complex $\Omega^{\bullet}\langle Y\rangle^{(D)}$ will be exact and therefore we have for $k>0$ that $\widetilde{\Omega}^{k}\langle Y\rangle^{(D)}=\operatorname{ker}\left(\Upsilon: \Omega^{k}\langle Y\rangle^{(D)} \rightarrow \Omega^{k-1}\langle Y\rangle^{(D-1)}\right)$.

For any $\tilde{\omega} \in \widetilde{\Omega}^{k}\langle Y\rangle^{(D)}$, the relations above imply that $d F \wedge \tilde{\omega} \in \widetilde{\Omega}^{k+1}\langle Y\rangle^{(D)}$ so that $\left(\widetilde{\Omega^{\bullet}}\langle Y\rangle^{(D)}, d F \wedge\right)$ defines a complex.

Similarly we get a third complex $\left(\widehat{\Omega}^{\bullet}\langle Y\rangle^{(D)}, \phi\right)$ by setting

$$
\widehat{\Omega}^{k}\langle Y\rangle^{(D)}=\widetilde{\Omega}^{k-1}\langle Y\rangle^{(D)},
$$

for any $k \geq 1$ and for $k=0$ (using the bigrading) set $\left(\widehat{\Omega}^{0}\langle Y\rangle\right)^{(D, J)}=0$. The boundary map defining this complex $\phi$ is given by $\phi(\hat{\omega})=-d_{\langle Y\rangle} F \wedge \hat{\omega}$ for $k \geq 1$ and $\phi=0$ for $k=0$.

We get a short exact sequence of complexes

$$
0 \longrightarrow \widetilde{\Omega}^{\bullet}\langle Y\rangle^{(D)} \longrightarrow \Omega^{\bullet}\langle Y\rangle^{(D)} \stackrel{\Upsilon^{\prime}}{\longrightarrow} \widehat{\Omega}^{\bullet}\langle Y\rangle^{(D)} \longrightarrow 0,
$$

where the arrow on the left is the inclusion and on the right we define $\Upsilon^{\prime}=\Upsilon$ for $k>0$, and for $k=0, \Upsilon^{\prime}$ is the zero map.

Letting $\Xi$ denote the connecting homomorphism we consider the corresponding long exact sequence in cohomology:

$$
\begin{aligned}
\ldots \stackrel{\Xi}{\longrightarrow} H^{k}\left(\widetilde{\Omega}^{\bullet}\langle Y\rangle^{(D)}\right) \stackrel{i}{\longrightarrow} H^{k}\left(\Omega^{\bullet}\langle Y\rangle^{(D)}\right) \stackrel{\Upsilon^{\prime}}{\longrightarrow} H^{k}\left(\widehat{\Omega}^{\bullet}\langle Y\rangle^{(D)}\right) \\
\stackrel{\Xi}{\longrightarrow} H^{k+1}\left(\widetilde{\Omega}^{\bullet}\langle Y\rangle^{(D)}\right) \stackrel{i}{\longrightarrow} \ldots
\end{aligned}
$$

Notice that since $\Xi$ is defined in terms of $\phi$ that it will shift the bidegree by $(0,1)$.

We can now substitute $H^{k}\left(\widehat{\Omega}^{\bullet}\langle Y\rangle\right)=H^{k-1}\left(\widetilde{\Omega}^{\bullet}\langle Y\rangle\right)$ for each $k \geq 1$. We have already verified that for every $J, H^{k}\left(\Omega^{\bullet}\langle Y\rangle\right)^{(D, J)}=0$, if $k \leq n+r-2$ and $k>n+r$. 
Therefore for each $J$ the long exact cohomology sequence reduces to

$$
\begin{aligned}
0 & \longrightarrow H^{n+r-1}\left(\widetilde{\Omega}^{\bullet}\langle Y\rangle\right)^{(D, J)} \longrightarrow H^{n+r-1}\left(\Omega^{\bullet}\langle Y\rangle\right)^{(D, J)} \\
& \longrightarrow H^{n+r-2}\left(\widetilde{\Omega}^{\bullet}\langle Y\rangle\right)^{(D, J)} \longrightarrow H^{n+r}\left(\widetilde{\Omega}^{\bullet}\langle Y\rangle\right)^{(D, J+1)} \\
& \longrightarrow H^{n+r}\left(\Omega^{\bullet}\langle Y\rangle\right)^{(D, J+1)} \longrightarrow H^{n+r-1}\left(\widetilde{\Omega}^{\bullet}\langle Y\rangle\right)^{(D, J+1)} \longrightarrow 0 .
\end{aligned}
$$

Since $\Upsilon\left(\Omega^{n+r-1}\right) \subseteq \Omega^{n+r-2}$ and $H^{n+r-2}\left(\Omega^{\bullet}\right)=0$, we have that the space $H^{n+r-2}\left(\widetilde{\Omega}^{\bullet}\langle Y\rangle\right)^{(D, J)}=0$. Also since $\widetilde{\Omega}^{n+r}=\Upsilon\left(\Omega^{n+r+1}\right)=0$, it follows that $H^{n+r}\left(\widetilde{\Omega}^{\bullet}\langle Y\rangle\right)^{(D, J+1)}=0$. This induces isomorphisms $H^{n+r-1}\left(\widetilde{\Omega}^{\bullet}\langle Y\rangle\right)^{(D, J)} \cong$ $H^{n+r-1}\left(\Omega^{\bullet}\langle Y\rangle\right)^{(D, J)}$ and $H^{n+r-1}\left(\widetilde{\Omega}^{\bullet}\langle Y\rangle\right)^{(D, J)} \cong H^{n+r}\left(\Omega^{\bullet}\langle Y\rangle\right)^{(D, J)}$

For the proof of the last assertion we take a form in $H^{n+r}\left(\Omega\langle Y\rangle^{(D, J)}\right)$ which will be a sum of elements from spaces

$$
\left(y_{1}^{v_{1}} \ldots y_{r}^{v_{r}}\right)\left(\mathbb{F}_{q}[x] /\left(f_{i_{1}}, \ldots, f_{i_{k}}\right)\right)^{\left(D+\sum d_{j} v_{j}-n, J\right)}\left(d x_{i}\right)_{i=1}^{n}\left(d y_{j} / y_{j}\right)_{j=1}^{r} .
$$

The degree of the Hilbert polynomial of this space uses the degrees in the quotient for which the corresponding $v_{j}$ 's are not zero, that is, $\sum_{j=1}^{\kappa}\left(d_{j}-1\right)=\sum_{j=1}^{\kappa} d_{j}-\kappa$. Now we need to find $J$ so that

$$
D+\sum_{j=1}^{\kappa} d_{j} v_{j}-n>\sum_{j=1}^{\kappa} d_{j}-\kappa .
$$

Any quotient space with such a degree would be zero. Moving the sum on the right and combining with the left leaves $D+\sum_{j=1}^{\kappa} d_{j}\left(v_{j}-1\right)-n$, which is greater than or equal to $D+\sum_{j=1}^{\kappa}\left(v_{j}-1\right)-n$ since each degree $d_{j}$ is positive. So substituting $J=\sum_{j=1}^{\kappa} v_{j}$ we have

$$
D+J-\kappa-n>-\kappa,
$$

which is true whenever $D+J>n$, that is, whenever $J \geq n$.

3.5. Bases for cohomology spaces. In this section we show that the computations in characteristic $p$, from the previous sections, completely determine the cohomology of the original complex $\Omega_{C(b, q-1, \delta)}^{\bullet}\langle Y\rangle^{(0)}$ in characteristic zero.

For $J=0, \ldots, n-1$ let $\mu_{J}$ be the dimension of $H^{n+r}\left(\Omega_{\mathbb{F}_{q}[x, y] / \mathbb{F}_{q}}\langle Y\rangle\right)^{(D, J)}$ as above. Choose integers $u_{i}(J, k)$ and $v_{j}(J, k)$ so that for $1 \leq k \leq \mu_{J}$, representatives of each cohomology class can be given by the monomial forms

$$
\bar{\xi}_{k}^{(J)}(d x)\left(\frac{d y}{y}\right)=\prod_{i=1}^{n} x_{i}^{u_{i}(J, k)} \prod_{j=1}^{r} y_{j}^{v_{j}(J, k)} d x_{1} \ldots d x_{n} \frac{d y_{1}}{y_{1}} \ldots \frac{d y_{r}}{y_{r}} .
$$

Now define $\xi_{k}^{(J)}=\pi^{(p-1) b J} \prod_{i=1}^{n} x_{i}^{u_{i}(J, k)} \prod_{j=1}^{r} y_{j}^{v_{j}(J, k)}$ to be the lifting of the $\bar{\xi}_{k}^{(J)}$ into characteristic zero. With the factor of $\pi^{(p-1) b J}$ these elements will be in $\mathcal{F}^{0} C(b, q-1, \delta)^{(D)}$. Also these monomials will be the coefficients in the preimage of $\bar{\xi}_{k}^{(J)}$ under the map (3-5).

Proposition 3.9. For $k=1, \ldots, \mu_{J}$ and $J=0, \ldots, n-1$, the classes $\left[\xi_{k}^{(J)}\right]$ generate a $\Gamma_{0}$-basis for $H^{n+r}\left(\Omega_{C(b, q-1, \delta)}^{\bullet}\langle Y\rangle\right)^{(D)}$.

Proof. The classes $\left[\xi_{k}^{(J)}\right]$ are linearly independent over $\Gamma_{0}$. If not, we can suppose there are $a_{k, J} \in \Gamma_{0}$ and $\eta \in \Omega_{C(b, q-1, \delta)}^{n+r-2}\langle Y\rangle^{(D)}$ such that $\sum_{k, J} a_{k, J} \Upsilon\left(\xi_{k}^{(J)}\right)=$ 
$\pi^{(p-1) b} \gamma^{-1} \Delta(\eta)$. Multiplying by an appropriate power of $\pi$ if needed, we can further assume that the $a_{k, J} \in \mathcal{O}_{\Gamma_{0}}$ with at least one $a_{k, J} \notin \pi \mathcal{O}_{\Gamma_{0}}$.

If $\eta \notin \mathcal{F}^{0} \Omega_{C(b, q-1, \delta)}^{n+r-2}\langle Y\rangle^{(D)}$, choose $s>0$ so that $\eta \in \mathcal{F}^{-s} \Omega_{C(b, q-1, \delta)}^{n+r-2}\langle Y\rangle^{(D)}$. Now by the relation $\sum_{k, J} a_{k, J} \Upsilon\left(\xi_{k}^{(J)}\right)=\pi^{(p-1) b} \gamma^{-1} \Delta(\eta)$ we have that $\pi^{(p-1) b} \gamma^{-1} \Delta\left(\pi^{s} \eta\right)$ $\in \mathcal{F}^{1} \Omega_{C(b, q-1, \delta)}^{n+r-2}\langle Y\rangle^{(D)}$. So by the reduction map (3-5) $d_{\langle Y\rangle}(F) \wedge \overline{\pi^{s} \eta}=0$.

Since $H^{n+r-2}\left(\Omega_{\mathbb{F}_{q}[x] / \mathbb{F}_{q}}\langle Y\rangle^{(D)}\right)=0$, there exists $\zeta \in \Omega_{\mathbb{F}_{q}[x] / \mathbb{F}_{q}}^{n+r-3}\langle Y\rangle^{(D)}$ such that $\overline{\pi^{s} \eta}=d_{\langle Y\rangle}(F) \wedge \zeta$. Now choose a lifting $\hat{\zeta} \in \mathcal{F}^{0} \Omega_{C(b, q-1, \delta)}^{n+r-3}\langle Y\rangle^{(D)}$. Then $\pi^{s} \eta-$ $\pi^{(p-1) b} \gamma^{-1} \Delta(\hat{\zeta}) \in \mathcal{F}^{1} \Omega_{C(b, q-1, \delta)}^{n+r-3}\langle Y\rangle^{(D)}$, so that $\eta_{1}=\eta-\pi^{(p-1) b} \gamma^{-1} \Delta\left(\pi^{-s} \hat{\zeta}\right) \in$ $\mathcal{F}^{-(s-1)} \Omega_{C(b, q-1, \delta)}^{n+r-2}\langle Y\rangle^{(D)}$. Notice that $\pi^{(p-1) b} \gamma^{-1} \Delta\left(\eta_{1}\right)=\pi^{(p-1) b} \gamma^{-1} \Delta(\eta)$ and in particular that $\sum_{k, J} a_{k, J} \Upsilon\left(\xi_{k}^{(J)}\right)=\pi^{(p-1) b} \gamma^{-1} \Delta\left(\eta_{1}\right)$.

We can repeat this process inductively and reduce $s$ to 0 , so that we may assume the above relation with $\eta_{1} \in \mathcal{F}^{0} \Omega_{C(b, q-1, \delta)}^{n+r-2}\langle Y\rangle^{(D)}$.

It follows using (3-5) that $\sum_{k, J} \bar{a}_{k, J} \Upsilon\left(\bar{\xi}_{k}^{(J)}\right)=d_{\langle Y\rangle}(F) \wedge \bar{\eta}$, with at least one $\bar{a}_{k, J} \neq 0$. Therefore the set of elements $\Upsilon\left(\bar{\xi}_{k}^{(J)}\right)$ are linearly dependent in the space $H^{n+r-1}\left(\Omega_{\mathbb{F}_{q}[x] / \mathbb{F}_{q}}\langle Y\rangle^{(D)}\right)$. However, the set of $\bar{\xi}_{k}^{(J)}$ are linearly independent in the space $H^{n+r}\left(\Omega_{\mathbb{F}_{q}[x] / \mathbb{F}_{q}}\langle Y\rangle^{(D)}\right)$ and $\Upsilon$ is an isomorphism, which gives a contradiction. This contradiction shows that the set of $\Upsilon\left(\left[\xi_{k}^{(J)}\right]\right)$ are linearly independent in the space $H^{n+r-1}\left(\Omega_{C(b, q-1, \delta)}^{\bullet}\langle Y\rangle^{(D)}\right)$; hence the set of $\left[\xi_{k}^{(J)}\right]$ must be linearly independent in $H^{n+r}\left(\Omega_{C(b, q-1, \delta)}^{\bullet}\langle Y\rangle^{(D)}\right)$.

To show that the set $\left\{\left[\xi_{k}^{(J)}\right]: 1 \leq k \leq \mu_{J}, 0 \leq J \leq n-1\right\}$ spans the space $H^{n+r}\left(\Omega_{C(b, q-1, \delta)}^{\bullet}\langle Y\rangle\right)^{(D)}$, we take $\eta \in \mathcal{F}^{0} \Omega_{C(b, q-1, \delta)}^{n+r}\langle Y\rangle^{(D)}$ and show that it must be of the form

$$
\eta=\sum_{i, j} c_{i, j} \prod_{i=1}^{n} x_{i}^{u_{i}(J, k)} \prod_{j=1}^{r} y_{j}^{v_{j}(J, k)} d x_{1} \ldots d x_{n} \frac{d y_{1}}{y_{1}} \ldots \frac{d y_{r}}{y_{r}}+\pi^{(p-1) b} \gamma^{-1} \Delta(\zeta)
$$

for some $c_{i, j} \in \widetilde{\Gamma}_{0}$ and $\zeta \in \mathcal{F}^{0} \Omega_{C(b, q-1, \delta)}^{n+r-1}\langle Y\rangle^{(D)}$. Using the isomorphism in (3-5) we can write

$\eta=\sum_{i, j} c_{i, j, 1} \prod_{i=1}^{n} x_{i}^{u_{i}(J, k)} \prod_{j=1}^{r} y_{j}^{v_{j}(J, k)} d x_{1} \ldots d x_{n} \frac{d y_{1}}{y_{1}} \ldots \frac{d y_{r}}{y_{r}}+\pi \eta_{1}+\pi^{(p-1) b} \gamma^{-1} \Delta\left(\zeta_{1}\right)$,

where $c_{i, j, 1} \in \widetilde{\Gamma}_{0}$, and $c_{i, j, 1} \prod x_{i}^{u_{i}} \prod y_{j}^{v_{j}} \in \mathcal{F}^{0} C(b, d, \delta)^{(0)} ;$ also $\eta_{1} \in \mathcal{F}^{0} \Omega_{C(b, d, \delta)}^{n+r}\langle Y\rangle^{(D)}$ and $\zeta_{1} \in \mathcal{F}^{0} \Omega_{C(b, d, \delta)}^{n+r-1}\langle Y\rangle^{(D)}$.

Suppose we can also write

$\eta=\sum_{i, j} c_{i, j, s} \prod_{i=1}^{n} x_{i}^{u_{i}(J, k)} \prod_{j=1}^{r} y_{j}^{v_{j}(J, k)} d x_{1} \ldots d x_{n} \frac{d y_{1}}{y_{1}} \ldots \frac{d y_{r}}{y_{r}}+\pi^{s} \eta_{s}+\pi^{(p-1) b} \gamma^{-1} \Delta\left(\zeta_{s}\right)$, 
where each $c_{i, j, s} \in \widetilde{\Gamma}_{0}$, and $c_{i, j, s} \prod x_{i}^{u_{i}} \prod y_{j}^{v_{j}} \in \mathcal{F}^{0} C(b, q-1, \delta)^{(0)} ;$ also $\eta_{s} \in$ $\mathcal{F}^{0} \Omega_{C(b, q-1, \delta)}^{n+r}\langle Y\rangle^{(D)}$ and $\zeta_{s} \in \mathcal{F}^{0} \Omega_{C(b, q-1, \delta)}^{n+r-1}\langle Y\rangle^{(D)}$ such that

$$
\left(c_{i, j, s}-c_{i, j, s-1}\right) \prod_{i=1}^{n} x_{i}^{u_{i}(J, k)} \prod_{j=1}^{r} y_{j}^{v_{j}(J, k)} \in \mathcal{F}^{s-1} \Omega_{C(b, q-1, \delta)}^{n+r}\langle Y\rangle^{(D)}
$$

and $\zeta_{s}-\zeta_{s-1} \in \mathcal{F}^{s-1} \Omega_{C(b, q-1, \delta)}^{n+r-1}\langle Y\rangle^{(D)}$.

We can also write for any $s \geq 1$,

$\eta_{s}=\sum_{i, j} c_{i, j}^{\prime} \prod_{i=1}^{n} x_{i}^{u_{i}(J, k)} \prod_{j=1}^{r} y_{j}^{v_{j}(J, k)} d x_{1} \ldots d x_{n} \frac{d y_{1}}{y_{1}} \ldots \frac{d y_{r}}{y_{r}}+\pi \eta_{s+1}+\pi^{(p-1) b} \gamma^{-1} \Delta\left(\zeta_{s}^{\prime}\right)$,

with $c_{i, j}^{\prime} \in \widetilde{\Gamma}_{0}$, and $c_{i, j}^{\prime} \prod x_{i}^{u_{i}} \prod y_{j}^{v_{j}} \in \mathcal{F}^{0} C(b, q-1, \delta)$; also we have $\eta_{s+1} \in$ $\mathcal{F}^{0} \Omega_{C(b, q-1, \delta)}^{n+r}\langle Y\rangle^{(D)}$ and $\zeta_{s}^{\prime} \in \mathcal{F}^{0} \Omega_{C(b, q-1, \delta)}^{n+r-1}\langle Y\rangle^{(D)}$.

If we set $c_{i, j, s+1}=c_{i, j, s}+\pi^{s} c_{i, j}^{\prime}$ and $\zeta_{s+1}=\zeta_{s}+\pi^{s} \zeta_{s}^{\prime}$, then substituting into (3-31) we have

$$
\begin{aligned}
\eta & =\sum_{i, j} c_{i, j, s+1} \prod_{i=1}^{n} x_{i}^{u_{i}(J, k)} \prod_{j=1}^{r} y_{j}^{v_{j}(J, k)} d x_{1} \ldots d x_{n} \frac{d y_{1}}{y_{1}} \ldots \frac{d y_{r}}{y_{r}} \\
& +\pi^{(s+1)} \eta_{s+1}+\pi^{(p-1) b} \gamma^{-1} \Delta\left(\zeta_{s+1}\right) .
\end{aligned}
$$

Now if we let $c_{i, j}=\lim _{s \rightarrow \infty} c_{i, j, s} \in \Gamma_{0}$ and $\zeta=\lim _{s \rightarrow \infty} \zeta_{s} \in \mathcal{F}^{0} \Omega_{C(b, q-1, \delta)}^{n+r-1}\langle Y\rangle^{(D)}$, then we have

$$
\eta=\sum_{i, j} c_{i, j} \prod_{i=1}^{n} x_{i}^{u_{i}(J, k)} \prod_{j=1}^{r} y_{j}^{v_{j}(J, k)} d x_{1} \ldots d x_{n} \frac{d y_{1}}{y_{1}} \ldots \frac{d y_{r}}{y_{r}}+\pi^{(p-1) b} \gamma^{-1} \Delta(\zeta),
$$

which completes the proof.

If we now put the factor of $\prod_{j=1}^{r} y_{j}^{\delta_{j} /(q-1)}$ back in we won't have affected the cohomology, so we have that for $0 \leq J \leq n-1$ and $1 \leq k \leq \mu_{J}$,

$$
\begin{aligned}
\widehat{\xi}_{k}^{(J)}(d x)\left(\frac{d y}{y}\right)=\pi^{\frac{(p-1) b\left(\sum_{j=1}^{r} \delta_{j}\right)}{(q-1)}} \prod_{j=1}^{r} y_{j}^{\delta_{j} /(q-1)} \xi_{k}^{(J)}(d x)\left(\frac{d y}{y}\right) \\
=\pi^{\frac{(p-1) b\left(J(q-1)+\sum_{j=1}^{r} \delta_{j}\right)}{(q-1)}} \prod_{i=1}^{n} x_{i}^{u_{i}(J, k)} \prod_{j=1}^{r} y_{j}^{v_{j}(J, k)+\frac{\delta_{j}}{q-1}} d x_{1} \ldots d x_{n} \frac{d y_{1}}{y_{1}} \ldots \frac{d y_{r}}{y_{r}}
\end{aligned}
$$

form a basis for $H^{n+r}\left(\Omega_{C(b, q-1, \delta)}^{\bullet}\langle Y\rangle\right)^{(0)}$.

\section{Hilbert SERIES}

Grading by $\operatorname{deg}_{2}=J$, we can use combinatorial methods to get a formula for the Hilbert series of the Dwork complex $\Omega^{\bullet}\langle Y\rangle^{(D, \bullet)}$, which we use to then deduce a formula for the Hilbert series of the non-zero cohomology space of dimension $n+r$. This formula (4-15) depends on $q$, the degrees $d_{j}$ and the original choice of multiplicative characters.

We fix a choice of characters, that is, we fix a $\delta$, and we denote the Hilbert series for the $(n+r)$-dimensional cohomology by $H_{\delta}(t)$. It will follow from our formula that $H_{\delta}(t)$ is a polynomial of degree $n-1$, and we write $H_{\delta}(t)=\sum_{J=0}^{n-1} \mu_{J} t^{J}$, where $\mu_{J}=\operatorname{dim} H^{n+r}\left(\Omega_{\mathbb{F}_{q}[x, y] / \mathbb{F}_{q}}^{\bullet}\langle Y\rangle\right)^{(D, J)}$. 
Consider $\Omega^{\bullet}\langle Y\rangle^{(D, \bullet)}$ as the sum of complexes

$$
0 \longrightarrow \Omega^{0}\langle Y\rangle^{(D, J-n-r)} \longrightarrow \ldots \longrightarrow \Omega^{n+r}\langle Y\rangle^{(D, J)} \longrightarrow 0
$$

for $0 \leq J<\infty$. From the definition of $\operatorname{deg}_{2}$, it is clear that $\Omega^{k}\langle Y\rangle^{(D, J)}=0$ for any $k$ whenever $J<0$. We define the Hilbert series of $\Omega^{\bullet}\langle Y\rangle^{(D, \bullet)}$ as

$$
K(t)=\sum_{J=0}^{\infty}\left(\sum_{k=0}^{n+r}(-1)^{k+n+r} d(k, J)\right) t^{J}
$$

where $d(k, J)=\operatorname{dim} \Omega^{k}\langle Y\rangle^{(D, J+k-n-r)}$, the dimension of the $k$ th element in the $J$ th row in (4-1).

By definition $\Omega^{k}\langle Y\rangle^{(D, J)}$ is a sum of spaces of the form

$$
\mathbb{F}_{q}[x, y]^{(D-\ell)} d x_{i_{1}} \ldots d x_{i_{\ell}} \frac{d y_{j_{1}}}{y_{j_{1}}} \ldots \frac{d y_{j_{m}}}{y_{j_{m}}} .
$$

Therefore, we start by counting elements in a canonical basis for such spaces given by forms of the type $x_{1}^{u_{1}} \ldots x_{n}^{u_{n}} y_{1}^{v_{1}} \ldots y_{r}^{v_{r}} d x_{i_{1}} \ldots d x_{i_{\ell}} \frac{d y_{j_{1}}}{y_{j_{1}}} \ldots \frac{d y_{j_{m}}}{y_{j_{m}}}$. The restriction on $\operatorname{deg}_{1}$ gives $D=u_{1}+\cdots+u_{n}-d_{1} v_{1}-\cdots-d_{r} v_{r}+\ell$, and for $\operatorname{deg}_{2}$ we have $J=v_{1}+\cdots+v_{r}$.

Fixing $v_{1}, \ldots, v_{r}$ and $\ell$, we have that the number of sets $\left\{u_{1}, \ldots, u_{n}\right\}$ satisfying the condition on $\operatorname{deg}_{1}$ is given by the binomial coefficient

$$
\left(\begin{array}{c}
d_{1} v_{1}+\cdots+d_{r} v_{r}-\ell+D+n-1 \\
n-1
\end{array}\right) .
$$

Defining the polynomial

$$
P_{\ell}\left(v_{1}, \ldots, v_{r}\right)=\frac{1}{(n-1) !} \prod_{k=1}^{n-1}\left(d_{1} v_{1}+\cdots+d_{r} v_{r}-\ell+D+k\right),
$$

it is clear that

$$
P_{\ell}\left(v_{1}, \ldots, v_{r}\right)=\left(\begin{array}{c}
d_{1} v_{1}+\cdots+d_{r} v_{r}-\ell+D+n-1 \\
n-1
\end{array}\right) .
$$

Now consider the series in $r$ variables, generated by $P_{\ell}\left(v_{1}, \ldots, v_{r}\right)$, associated to the index $j_{1}, \ldots, j_{m}$,

$$
K_{\ell}\left(j_{1}, \ldots, j_{m} ; t_{1}, \ldots, t_{r}\right)=\sum_{v_{1}, \ldots, v_{r}=0}^{\infty} P_{\ell}\left(v_{1}, \ldots, v_{r}\right) t_{1}^{v_{1}} \ldots t_{r}^{v_{r}} .
$$

Therefore for fixed $\ell$ and fixed $j_{1}, \ldots, j_{m}$, by setting each $t_{j}=t$ we have

$$
K_{\ell}\left(j_{1}, \ldots, j_{m} ; t, \ldots, t\right)=\sum_{J=0}^{\infty}\left(\operatorname{dim}_{\mathbb{F}_{q}}\left(\mathbb{F}_{q}[x, y] d x_{i_{1}} \ldots d x_{i_{\ell}} \frac{d y_{j_{1}}}{y_{j_{1}}} \ldots \frac{d y_{j_{m}}}{y_{j_{m}}}\right)^{(D, J)}\right) t^{J}
$$

Summing down the columns of (4-1) we can now use (4-5) to write

$$
K(t)=\sum_{\ell=0}^{n} \sum_{m=0}^{r} \sum_{\left\{1 \leq j_{1}<\cdots<j_{m} \leq r\right\}}(-1)^{n+r-\ell-m}\left(\begin{array}{l}
n \\
\ell
\end{array}\right) t^{n+r-\ell-m} K_{\ell}\left(j_{1}, \ldots, j_{m} ; t, \ldots, t\right) .
$$


Note that for any set of indices of length $m$, we have $K_{\ell}\left(j_{1}, \ldots, j_{m} ; t, \ldots, t\right)=$ $K_{\ell}\left(j_{1}^{\prime}, \ldots, j_{m}^{\prime} ; t, \ldots, t\right)$ for all $m=0, \ldots, r$. Therefore we can simplify (4-6) slightly, by setting $K_{\ell}\left(m ; t_{1}, \ldots, t_{r}\right)=K_{\ell}\left(j_{1}, \ldots, j_{m} ; t_{1}, \ldots, t_{r}\right)$ for each $m$, which yields

$$
K(t)=\sum_{\ell=0}^{n} \sum_{m=0}^{r}(-1)^{n+r-\ell-m}\left(\begin{array}{l}
n \\
\ell
\end{array}\right)\left(\begin{array}{c}
r \\
m
\end{array}\right) t^{n+r-\ell-m} K_{\ell}(m ; t, \ldots, t) .
$$

Since $P_{\ell}$ is a polynomial, in $v_{1}, \ldots, v_{r}$, of degree $n-1$ we can write

$$
P_{\ell}\left(v_{1}, \ldots, v_{r}\right)=\sum_{e_{1}+\cdots+e_{r} \leq n-1} A_{\ell}\left(e_{1}, \ldots, e_{r}\right) v_{1}^{e_{1}} \ldots v_{r}^{e_{r}},
$$

for some rational coefficients $A_{\ell}\left(e_{1}, \ldots, e_{r}\right)$. If we set $E=e_{1}+\cdots+e_{r}$ and let $S_{i}\left(z_{1}, \ldots, z_{n-1}\right)$ denote the $i$ th elementary symmetric function in $n-1$ variables, then one can prove (by induction on $r$ ) that these coefficients satisfy

$$
\begin{aligned}
& A_{\ell}\left(e_{1}, \ldots, e_{r}\right)= \\
& \quad \frac{(-1)^{n-1-E} E !}{(n-1) ! e_{1} ! \ldots e_{r} !} S_{n-1-E}(\ell-D-(n-1), \ldots, \ell-D-1) d_{1}^{e_{1}} \ldots d_{r}^{e_{r}} .
\end{aligned}
$$

Making this substitution we can now write

$$
K_{\ell}\left(m ; t_{1}, \ldots, t_{r}\right)=\sum_{E \leq n-1} A_{\ell}\left(e_{1}, \ldots, e_{r}\right) \sum_{v_{1}, \ldots, v_{r}=0}^{\infty} v_{1}^{e_{1}} \ldots v_{r}^{e_{r}} t_{1}^{v_{1}} \ldots t_{r}^{v_{r}} .
$$

If we define the polynomial $Q_{e}(t)$ by the relation on Taylor series

$$
\begin{aligned}
\sum_{v=0}^{\infty} v^{e} t^{v} & =\left(t \frac{d}{d t}\right)^{e} \frac{1}{1-t} \\
& =\frac{Q_{e}(t)}{(1-t)^{e+1}},
\end{aligned}
$$

then we can rewrite (4-10) as

$$
K_{\ell}(m ; t, \ldots, t)=\sum_{E \leq n-1} A_{\ell}\left(e_{1}, \ldots, e_{r}\right) \frac{Q_{e_{1}}(t) \ldots Q_{e_{r}}(t)}{(1-t)^{E+r}} .
$$

Using the binomial coefficients from the expansion of $(1-t)^{r}$ and fixing $e_{1}, \ldots, e_{r}$ we also have

$$
\sum_{m=0}^{r}(-1)^{r-m}\left(\begin{array}{c}
r \\
m
\end{array}\right) t^{r-m} Q_{e_{1}}(t) \ldots Q_{e_{r}}(t)=(1-t)^{r} Q_{e_{1}}(t) \ldots Q_{e_{r}}(t) .
$$

These substitutions reduce the Hilbert series of $\Omega^{\bullet}\langle Y\rangle^{(D, \bullet)}$ to

$$
K(t)=\sum_{\ell=0}^{n}(-1)^{n-\ell}\left(\begin{array}{l}
n \\
\ell
\end{array}\right) t^{n-\ell} \sum_{E \leq n-1} A_{\ell}\left(e_{1}, \ldots, e_{r}\right) \frac{Q_{e_{1}}(t) \ldots Q_{e_{r}}(t)}{(1-t)^{E}} .
$$

Now from vanishing of the cohomology except for dimensions $n+r-1$ and $n+r$, we have

$$
\begin{aligned}
\sum_{k=0}^{n+r}(-1)^{k} \operatorname{dim} \Omega^{k}\langle Y\rangle^{(D, \bullet)}= & \sum_{k=0}^{n+r}(-1)^{k} \operatorname{dim} H^{k}\left(\Omega^{\bullet}\langle Y\rangle^{(D, \bullet)}\right) \\
= & (-1)^{n+r-1} \operatorname{dim} H^{n+r-1}\left(\Omega^{\bullet}\langle Y\rangle^{(D, \bullet)}\right) \\
& +(-1)^{n+r} \operatorname{dim} H^{n+r}\left(\Omega^{\bullet}\langle Y\rangle^{(D, \bullet)}\right) .
\end{aligned}
$$


In particular for each $J$,

$$
\begin{aligned}
\sum_{k=0}^{n+r}(-1)^{k} \operatorname{dim} \Omega^{k}\langle Y\rangle^{(D, J+k-n-r)}= & (-1)^{n+r} \sum_{k=0}^{n+r}(-1)^{k} d(k, J) \\
= & (-1)^{n+r-1} \operatorname{dim} H^{n+r-1}\left(\Omega^{\bullet}\langle Y\rangle^{(D, J-1)}\right) \\
& +(-1)^{n+r} \operatorname{dim} H^{n+r}\left(\Omega^{\bullet}\langle Y\rangle^{(D, J)}\right) .
\end{aligned}
$$

It follows that $K(t)=(1-t) H_{\delta}(t)$. So by (4-14) we have that

$$
\begin{aligned}
H_{\delta}(t) & =\sum_{\ell=0}^{n}(-1)^{n-\ell}\left(\begin{array}{c}
n \\
\ell
\end{array}\right) t^{n-\ell} \sum_{E \leq n-1} A_{\ell}\left(e_{1}, \ldots, e_{r}\right) \frac{Q_{e_{1}}(t) \ldots Q_{e_{r}}(t)}{(1-t)^{E+1}} \\
& =\sum_{E \leq n-1}\left(\sum_{\ell=0}^{n}(-1)^{n-\ell}\left(\begin{array}{c}
n \\
\ell
\end{array}\right) A_{\ell}\left(e_{1}, \ldots, e_{r}\right) t^{n-\ell}\right) \frac{Q_{e_{1}}(t) \ldots Q_{e_{r}}(t)}{(1-t)^{E+1}} .
\end{aligned}
$$

Using (4-15) we can also develop a related formula for $H_{\delta}(1)$ that gives the degree of the $L$-function associated to the sum (1-6) and will be independent of $\delta$. Define the polynomial $\widetilde{A}_{e_{1}, \ldots, e_{r}}(t)$ by

$$
\widetilde{A}_{e_{1}, \ldots, e_{r}}(t)=\sum_{\ell=0}^{n}(-1)^{n-\ell}\left(\begin{array}{l}
n \\
\ell
\end{array}\right) A_{\ell}\left(e_{1}, \ldots, e_{r}\right) t^{n-\ell},
$$

which simplifies $H_{\delta}(t)$ to

$$
H_{\delta}(t)=\sum_{E \leq n-1} \widetilde{A}_{e_{1}, \ldots, e_{r}}(t) \frac{Q_{e_{1}}(t) \ldots Q_{e_{r}}(t)}{(1-t)^{E+1}} .
$$

Now from the definitions of $A_{\ell}\left(e_{1}, \ldots, e_{r}\right)$ and $\widetilde{A}_{e_{1}, \ldots, e_{r}}(t)$ we have that $(1-t)^{E+1}$ divides $\widetilde{A}_{e_{1}, \ldots, e_{r}}(t)$. Indeed, consider $A_{\ell}\left(e_{1}, \ldots, e_{r}\right)$ as a polynomial in the variable $\ell$ of degree $n-1-E$. We can therefore write $\widetilde{A}_{e_{1}, \ldots, e_{r}}(t)$ as a linear combination of the polynomials

$$
\sum_{k=0}^{n}(-1)^{k}\left(\begin{array}{l}
n \\
k
\end{array}\right) k^{i} t^{k}=\left(t \frac{d}{d t}\right)^{i}(1-t)^{n}
$$

for $i=0, \ldots, n-1-E$, which are all divisible by $(1-t)^{E+1}$.

By Taylor's formula the value at $t=1$ of $\widetilde{A}_{e_{1}, \ldots, e_{r}}(t) /(1-t)^{E+1}$ is equal to the value at $t=1$ of

$$
\frac{(-1)^{E+1}}{(E+1) !}\left(\frac{d}{d t}\right)^{E+1} \widetilde{A}_{e_{1}, \ldots, e_{r}}(t),
$$

which is equal to the value at $t=1$ of

$$
\frac{(-1)^{E+1}}{(E+1) !}\left(t \frac{d}{d t}\right)^{E+1} \widetilde{A}_{e_{1}, \ldots, e_{r}}(t) .
$$

Similarly, the polynomial $\left(t \frac{d}{d t}\right)^{E+1} \widetilde{A}_{e_{1}, \ldots, e_{r}}(t)$ can be written as a linear combination of the polynomials in (4-16) for $i=E+1, \ldots, n$. In this linear combination we compute the coefficient of $\left(t \frac{d}{d t}\right)^{n}(1-t)^{n}$ to be

$$
\frac{d_{1}^{e_{1}} \ldots d_{r}^{e_{r}}}{(n-1-E) ! e_{1} ! \ldots e_{r} !} \text {. }
$$


It is clear that the values at $t=1$ for the polynomials in (4-16) are all zero for $i<n$, while for $i=n$ the value at $t=1$ is $(-1)^{n} n$ !. Therefore the value at $t=1$ of $(4-17)$ is

$$
(-1)^{n+E+1}\left(\begin{array}{c}
n \\
E+1
\end{array}\right) \frac{d_{1}^{e_{1}} \ldots d_{r}^{e_{r}}}{e_{1} ! \ldots e_{r} !}
$$

Now, since $Q_{e}(1)=e$ ! it follows that

$$
H_{\delta}(1)=\sum_{E \leq n-1}(-1)^{n+E+1}\left(\begin{array}{c}
n \\
E+1
\end{array}\right) d_{1}^{e_{1}} \ldots d_{r}^{e_{r}}
$$

\section{Newton POLYGON OF $L(f, \chi ; t)$}

Recall that if $v_{j} \equiv \delta_{j}(\bmod q-1)$, then $p \delta_{j}^{\prime} \equiv v_{j}(\bmod q-1)$. Recursively, for $I=0, \ldots, a-1$, we define $\delta^{(I)}=\left(\delta_{1}^{(I)}, \ldots, \delta_{r}^{(I)}\right)=\left(\delta^{(I-1)}\right)^{\prime}$ so that $p^{I} \delta^{(I)} \equiv$ $v_{j}(\bmod q-1)$. For each combination of $\delta$ 's, i.e. for different combinations of multiplicative characters, the dimensions $\mu_{J}$ may be different and we define $\mu_{J}^{(I)}$ accordingly. Construct an array with $a$ rows and $H_{\delta}(1)$ columns. Row 1 will be associated to $\delta^{(0)}$, row 2 with $\delta^{(1)}$, and so on. Each row will have integer entries ranging from 0 to $n-1$, which are taken $\mu_{J}^{(I)}$ at a time and ranked in ascending order. For $1 \leq s \leq H_{\delta}(1)$, let $\bar{J}_{s}$ be the average of the entries in the $s$ th column.

Theorem 5.1. Let $f_{1}, \ldots, f_{r}$ be non-constant homogeneous polynomials with coefficients in $\mathbb{F}_{q}$ and with $d_{j}=\operatorname{deg} f_{j}$. Assume $\left(p, d_{j}\right)=1$ for each $j=1, \ldots, r$. If $\chi_{1}, \ldots, \chi_{r}$ are non-trivial multiplicative characters on $\mathbb{F}_{q}$ such that $\prod_{j=1}^{r} \chi_{j}^{d_{j}}=\chi_{0}$ and if the set of solutions to the equation $\prod_{j=1}^{r} f_{j}(x)=0$ defines a divisor with normal crossings, then the Newton polygon of the L-function in (1-3) (with respect to ord $_{q}$ ) sits on or above the Newton polygon of the polynomial

$$
\prod_{s=1}^{H_{\delta}(1)}\left(1-q^{\bar{J}_{s}} t\right)
$$

Proof. Applying Theorem 3.1 to (2-14) gives

$$
L\left(f, \chi, \psi, T^{*} ; t\right)=\prod_{k=n+r-1}^{n+r} \operatorname{det}\left(I-t \alpha_{k} \mid H^{k}\left(\Omega_{C(b, d, \delta)}^{\bullet}\langle Y\rangle, \Delta\right)\right)^{(-1)^{k+1}} .
$$

For the rest of this chapter we abbreviate the notation and let $\operatorname{det}\left(I-t \alpha_{n+r}\right)=$ $\operatorname{det}\left(I-t \alpha_{n+r} \mid H^{n+r}\left(\Omega_{C(b, q-1, \delta)}^{\bullet}\langle Y\rangle\right)^{(0)}\right)$, and we note that the $\operatorname{determinants} \operatorname{det}(I-$ $\left.t \alpha_{n+r-1}\right)$ and $\operatorname{det}\left(I-q t \alpha_{n+r}\right)$ are equal following the method of [6], Theorem 6.16.

We will analyze $\alpha_{n+r}$ using the related operator $\beta_{n+r}$. The appearance of $\Psi$, in the definition of $\beta$, will have the effect of permuting the spaces $C(b, q-1, \delta)$ indexed by certain $\delta$ 's. As a result, we need to consider $a$ separate maps of the type $\beta: C(b, q-1, \delta)^{(0)} \longrightarrow C\left(b, q-1, \delta^{\prime}\right)^{(0)}$. This induces the map $\beta_{n+r}$ : $\Omega_{C\left(b, q-1, \delta^{(I)}\right)}^{n+r}\langle Y\rangle^{(0)} \longrightarrow \Omega_{C\left(b, q-1, \delta^{(I+1)}\right)}^{n+r}\langle Y\rangle^{(0)}$. For a fixed, acceptable choice of characters $\chi_{1}, \ldots, \chi_{r}$ we will denote the associated cohomology $H^{n+r}(\delta)$, where $\delta$ represents the $r$-tuple $\delta_{1}, \ldots, \delta_{r}$ as before. Now in general, for $I=1, \ldots, a-1$, as $\beta_{n+r}$ cycles through the spaces $C\left(b, q-1, \delta^{(I)}\right)$ we will write $\beta_{n+r}: H^{n+r}\left(\delta^{(I)}\right) \longrightarrow$ $H^{n+r}\left(\delta^{(I+1)}\right)$, where it is understood that $\delta=\delta^{(0)}$. It follows that $\beta_{n+r}$ is an endomorphism on the space $\mathbf{H}^{n+r}=\bigoplus_{I=0}^{a-1} H^{n+r}\left(\delta^{(I)}\right)$, and also that the map $\alpha_{n+r}$ 
is both a $\widetilde{\Gamma}_{0}$-linear and $\widetilde{\Gamma}_{1}$-linear endomorphism of $\mathbf{H}^{n+r}$ and also of each of the spaces $H^{n+r}\left(\delta^{(I)}\right)$.

With notation similar to Section 3.5, for $0 \leq I \leq a-1,0 \leq J \leq n-1$ and $1 \leq k \leq \mu_{J}^{(I)}$ we let $u_{i}$ and $v_{j}$ be integers depending on $k, J$ and $I$ so that if

$$
\xi_{k, I}^{(J)}=\prod_{i=1}^{n} x_{i}^{u_{i}} \prod_{j=1}^{r} y_{j}^{v_{j}+\delta_{j}^{(I)} /(q-1)},
$$

then the forms $\xi_{k, I}^{(J)} d x_{1} \ldots d x_{n} \frac{d y_{1}}{y_{1}} \ldots \frac{d y_{r}}{y_{r}}$ will generate a $\Gamma_{0}$-basis for each of the $H^{n+r}\left(\delta^{(I)}\right)$. We normalize each of these monomials with a factor of the form $\pi^{\epsilon}$ so that $\pi^{\epsilon} \xi_{k, I}^{(J)} \in \mathcal{F}^{0} C\left(b / p, q-1, \delta^{(I)}\right)^{(0)}$. Since $\beta=\Psi \circ \tau^{-1} \circ G$ maps $C\left(b / p, q-1, \delta^{(I)}\right)$ into $C\left(b, q-1, \delta^{(I+1)}\right)$ and since $\beta$ is made up of homogeneous elements, it will map $\mathcal{F}^{0} C\left(b / p, q-1, \delta^{(I)}\right)$ to $\mathcal{F}^{0} C\left(b, q-1, \delta^{(I+1)}\right)$.

We choose a basis $\gamma_{1}, \ldots, \gamma_{a}$ for $\widetilde{\Gamma}_{0}$ over $\widetilde{\Gamma}_{1}$ satisfying $p$-adic directness (see [9], Section 3c), that is, for any $c_{1}, \ldots, c_{a} \in \widetilde{\Gamma}_{1}, \operatorname{ord}_{p} \sum_{s=1}^{a} c_{s} \gamma_{s}=\min \left\{\operatorname{ord}_{p} c_{s}\right\}$.

It follows that the classes of $\gamma_{s} \pi^{\epsilon} \xi_{k, I}^{(J)}$ will form a $\widetilde{\Gamma}_{1}$-basis for $H^{n+r}\left(\chi, \delta^{(I)}\right)$. We denote the $a \cdot H_{\delta}(1) \times a \cdot H_{\delta}(1)$ matrix for $\beta_{n+r}: H^{n+r}\left(\chi, \delta^{(I)}\right) \longrightarrow H^{n+r}\left(\chi, \delta^{(I+1)}\right)$ by $[\mathbf{B}(I ; I+1)]$, with entries $\mathbf{B}\left(s, u, v, I ; s^{\prime}, u^{\prime}, v^{\prime}, I+1\right)$, where (dropping the $d x_{1} \wedge$ $\left.\cdots \wedge d x_{n} \wedge \frac{d y_{1}}{y_{1}} \wedge \cdots \wedge \frac{d y_{r}}{y_{r}}\right)$

$$
\beta_{n+r}\left(\gamma_{s} \pi^{\epsilon} \xi_{k, I}^{(J)}\right)=\sum_{s^{\prime}, k^{\prime}, J^{\prime}} \mathbf{B}\left(s, u, v, I ; s^{\prime}, u^{\prime}, v^{\prime}, I+1\right) \gamma_{s^{\prime}} \pi^{\epsilon^{\prime}} \xi_{k^{\prime}, I+1}^{\left(J^{\prime}\right)} .
$$

These $a$ matricies will form the only non-zero blocks of the matrix of the $\widetilde{\Gamma}_{1}$ endomorphism $\beta_{n+r}: \mathbf{H}^{n+r} \longrightarrow \mathbf{H}^{n+r}$, which takes the form

$$
\mathbf{B}=\left[\begin{array}{ccccc}
0 & 0 & \ldots & \ldots & {[\mathbf{B}(a-1 ; 0)]} \\
{[\mathbf{B}(0 ; 1)]} & 0 & & & 0 \\
\vdots & {[\mathbf{B}(1 ; 2)]} & & & \vdots \\
\vdots & \vdots & \ddots & \vdots & \vdots \\
0 & 0 & \ldots & {[\mathbf{B}(a-2 ; a-1)]} & 0
\end{array}\right] .
$$

It is straightforward to show that $\mathbf{B}^{a}$ is of the form

$$
\mathbf{B}^{a}=\left[\begin{array}{ccccc}
B_{1} & 0 & \ldots & \ldots & 0 \\
0 & B_{2} & & & 0 \\
\vdots & & B_{3} & & \vdots \\
\vdots & \vdots & \ddots & \vdots & \vdots \\
0 & 0 & \ldots & 0 & B_{a}
\end{array}\right]
$$

where the blocks down the diagonal are a product of all $a$ of the blocks $[B(I ; I+1)]$ in some order. The matrix for $\alpha_{n+r}: H^{n+r}(\chi, \delta) \rightarrow H^{n+r}(\chi, \delta)$, call it $\mathbf{A}$, which is equal to the block of $\mathbf{B}^{a}$ labeled $B_{1}$ in (5-4), is written

$$
\mathbf{A}=[\mathbf{B}(a-1 ; 0)] \cdot[\mathbf{B}(a-2 ; a-1)] \cdots \cdot[\mathbf{B}(1 ; 2)] \cdot[\mathbf{B}(0 ; 1)] .
$$

Write $\operatorname{det}(I-t \mathbf{B})=1+\sum_{k=1}^{N} b_{k} t^{k}$, where $N=a^{2} \cdot H_{\delta}(1)$ is the number of rows of $\mathbf{B}$. Recall that $b_{k}=0$ unless $a \mid k$, which says that $\operatorname{det}(I-t \mathbf{B})$ is actually a 
polynomial in $t^{a}$, so we can rewrite

$$
\operatorname{det}(I-t \mathbf{B})=\prod_{i=1}^{N / a}\left(1-\lambda_{i} t^{a}\right) .
$$

Now since the eigenvalues of $\mathbf{B}^{a}$ are just $a$ th powers of the eigenvalues of $\mathbf{B}$ we have

$$
\operatorname{det}\left(I-t \mathbf{B}^{a}\right)=\prod_{i=1}^{N / a}\left(1-\lambda_{i} t\right)^{a} .
$$

Also in $\mathbf{B}^{a}$ we see that each non-zero block down the diagonal is conjugate to $\mathbf{A}$ so that $[\operatorname{det}(I-t \mathbf{A})]^{a}=\operatorname{det}\left(I-t \prod_{j=1}^{a} B_{j}\right)$, where the product on the right is of the blocks of (5-4) in any acceptable order. Therefore $[\operatorname{det}(I-t \mathbf{A})]^{a}=\operatorname{det}\left(I-t \mathbf{B}^{a}\right)$, so that using (5-6) and (5-7) we get

$$
\begin{aligned}
\operatorname{det}(I-t \mathbf{A}) & =\operatorname{det}\left(I-\mathbf{B} t^{1 / a}\right) \\
& =\prod_{i=1}^{N / a}\left(1-\lambda_{i} t\right) \\
& =1+b_{a} t+\cdots+b_{N} t^{N / a} .
\end{aligned}
$$

From Section 5 of [17] we have the relation

$$
b_{m}=(-1)^{m} \sum \sum_{\sigma \in S_{m}} \operatorname{sign}(\sigma) \prod_{k=1}^{m} \mathbf{B}\left(j_{k}, u_{k}, v_{k} I ; j_{\sigma(k)}, u_{\sigma(k)}, v_{\sigma(k)}, I+1\right),
$$

where the outer sum is taken over all sets $\left\{\left(j_{k}, u_{k}, v_{k}\right)\right\}_{k=1}^{m}$ of $m$ distinct triples in the set $\{1, \ldots, a\} \times\left\{\bigcup_{I=0}^{a-1} H^{n+r}\left(\delta^{(I)}\right)\right\}$ (basis elements taken one at a time from each $H^{n+r}\left(\delta^{(I)}\right)$ in sets of size $a$ ), and the inner sum is taken over all permutations $\sigma$ on $m$ letters, with signature $\operatorname{sign}(\sigma)$. Moreover we may assume that $x^{u_{k}} y^{v_{k}} \in$ $H^{n+r}\left(\chi_{1}^{(I)}, \ldots, \chi_{r}^{(I)}\right)$ and $x^{u_{\sigma(k)}} y^{v_{\sigma(k)}} \in H^{n+r}\left(\chi_{1}^{(I+1)}, \ldots, \chi_{r}^{(I+1)}\right)$ for some $I$ and $k$.

Now the image of a basis element under $\beta_{n+r}$ will be in the filtered space $\mathcal{F}^{0} \Omega_{C\left(b, q-1, \delta^{(I+1)}\right)}^{n+r}\langle Y\rangle^{(0)}$, so it is a sum of terms $A\left(u^{\prime}, v^{\prime}\right) \pi^{\frac{(p-1) b\left|v^{\prime}\right|}{q-1}} x^{u^{\prime}} y^{v^{\prime}}$, where $A\left(u^{\prime}, v^{\prime}\right) \in \tilde{\pi}^{0} \mathcal{O}_{\tilde{\Gamma}_{0}}$. Therefore, using (5-2), the factor $\mathbf{B}\left(s, u, v, I: s^{\prime}, u^{\prime}, v^{\prime}, I+1\right) \pi^{\epsilon^{\prime}}$ will be divisible by $\pi \frac{(p-1) b\left[\sum v_{j}^{\prime}(q-1)+\delta_{j}^{(I+1)}\right]}{q-1}$, and therefore $\mathbf{B}\left(s, u, v, I ; s^{\prime}, u^{\prime}, v^{\prime}, I+1\right)$ is divisible by

$$
\pi^{\frac{(p-1) b\left[\sum v_{j}^{\prime}(q-1)+\delta_{j}^{(I+1)}\right]}{q-1}-\frac{(p-1) b\left[\sum v_{j}^{\prime}(q-1)+\delta_{j}^{(I+1)}\right]}{p(q-1)}}=\pi^{\frac{(p-1) b\left[\sum v_{j}^{\prime}(q-1)+\delta_{j}^{(I+1)}\right]}{q-1} \cdot\left(1-\frac{1}{p}\right) .}
$$

Now in each of the non-zero blocks $[\mathbf{B}(I ; I+1)]$, if we put the basis elements in ascending order ranked by $J$, then the first $a \mu_{0}^{(I)}$ rows will be divisible by $\pi^{\frac{(p-1) b\left[\sum \delta_{j}^{(I+1)}\right]}{q-1}(1-1 / p)}$, and the next $a \mu_{1}^{(I)}$ rows by $\pi^{\frac{(p-1) b\left[(q-1)+\sum \delta_{j}^{(I+1)}\right]}{q-1}(1-1 / p)}$, and

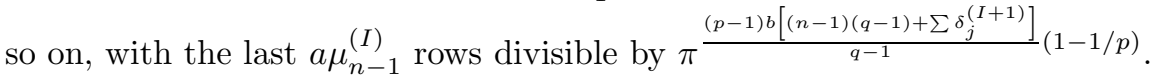

We construct an array with $a$ rows, corresponding to each $I=0, \ldots, a-1$ and $H_{\delta}(1)$ columns corresponding to the divisors of the $a \mu_{J}^{(I)}$ th row of the matrix $\mathbf{B}$. The first $\mu_{0}^{(0)}$ entries of row 1 are all equal to $\pi_{\frac{(p-1) b\left(0(q-1)+\sum \delta_{j}^{(I)}\right)}{q-1}(1-1 / p)}$, and the 
next $\mu_{1}^{(0)}$ entries are $\pi^{\frac{(p-1) b\left(1(q-1)+\sum \delta_{j}^{(I)}\right)}{q-1}(1-1 / p)}$, and so on, filling out the first row. The rest of the rows are constructed similarly. We note at this point that these values are independent of $b<p /(p-1)$ and we can simplify the array by taking the limit as $b \rightarrow p /(p-1)$, which will cancel out the factor of $(1-1 / p)$. This simplifies each of the entries to $\pi^{J+\sum \delta_{j}^{(I)} /(q-1)}$ for appropriate $J$ and $I$.

Simplify this array by taking just the exponent of $\pi$, so that the first $\mu_{0}^{(0)}$ entries of the first row are $0+\sum \delta_{j}^{(0)} /(q-1)$, the next $\mu_{1}^{(0)}$ entries of the first row are $1+\sum \delta_{j}^{(0)} /(q-1)$, and so on. For the entry in the $I$ th row and the $k$ th column, we label these entries $J(I, k)+\sum \delta_{j}^{(0)} /(q-1)$. In this case, the average down the $k$ th column is

$$
\frac{1}{a} \sum_{I=0}^{a-1}\left(J(I, k)+\sum_{j=1}^{r} \delta_{j}^{(I)} /(q-1)\right)=\frac{1}{a} \sum_{I=0}^{a-1} J(I, k)+\frac{1}{a} \sum_{I=0}^{a-1} \sum_{j=1}^{r} \delta_{j}^{(I)} /(q-1),
$$

for each $k=1, \ldots, H_{\delta}(1)$. From Stickelberger's theorem we see that the last term on the right is the $q$-ordinal of the product of Gauss sums in the formula for $T^{*}(f, \chi, \psi)$. We remove this factor from each entry of the array, so that the average down each column is reduced to $\bar{J}_{k}=\frac{1}{a} \sum_{I=0}^{a-1} J(I, k)$.

Since we now have a lower bound on the $q$-ordinal of each entry in the matrix, we can get a lower bound for each $b_{m}$ by taking $m$ columns of the array at a time. It follows from [3] that coordinates for the Newton polygon of $\operatorname{det}(I-t \mathbf{A})$ are given by $(0,0)$ and

$$
\left(\sum_{k=0}^{M} \bar{J}_{k}, \frac{1}{a} \sum_{k=0}^{M} k \bar{J}_{k}\right),
$$

$M=0,1, \ldots, n-1$. Since we have ordered the array as increasing from left to right, these vertices are the same as the vertices obtained by taking as the first coordinate pair $\left(1, \bar{J}_{1}\right)$, the second $\left(2, \bar{J}_{2}\right)$, and so on, which is equivalent to the statement in the theorem.

\section{EXAmples}

6.1. Stickelberger's theorem. Let $n=1$ and $r=1$. The space $\mathbb{P}^{0}$ consists of a single point, and the polynomial $f(x)=x^{d}$ satisfies the definition of a normal crossing divisor. The corresponding affine character sum is

$$
\sum_{x \in \mathbb{F}_{q}^{\times}} \chi\left(x^{d}\right)=q-1
$$

Since we are assuming that $\chi^{d}=\chi_{0}$, we must have $d \mid(q-1)$.

The related sum $T^{*}(f, \chi, \psi)$ gives

$$
\begin{aligned}
\sum_{x, y \in\left(\mathbb{F}_{q}\right)^{2}} \chi^{-1}(y) \psi\left(y x^{d}\right) & =g\left(\chi^{-1}, \psi\right) \cdot \sum_{x \in \mathbb{F}_{q}^{\times}} \chi\left(x^{d}\right) \\
& =(q-1) \cdot g\left(\chi^{-1}, \psi\right) .
\end{aligned}
$$

If we write $\chi=\widehat{\chi}^{\delta}$, where $\widehat{\chi}$ is the Teichmüller character on $\mathbb{F}_{q}$, then $1 \leq \delta \leq q-2$ and we can write $\delta=j_{0}+j_{1} p+\cdots+j_{a-1} p^{a-1}$ to define the integers $j_{0}, \ldots, j_{a-1}$. 
The estimates for $T^{*}$ give

$$
\begin{aligned}
\operatorname{ord}_{q}\left(g\left(\chi^{-1}, \psi\right)\right) & \geq \frac{1}{a}\left(\frac{\delta^{(0)}+\delta^{(1)}+\cdots+\delta^{(a-1)}}{q-1}\right) \\
& =\frac{j_{0}+j_{1}+\cdots+j_{a-1}}{a(p-1)} .
\end{aligned}
$$

Using the same formula with $(q-1)-\delta^{(I)}$ replacing $\delta^{(I)}$ we get the estimate

$$
\begin{aligned}
\operatorname{ord}_{q} g(\chi, \psi) & \geq \frac{1}{a} \sum_{I=0}^{a-1} \frac{(q-1)-\delta^{(I)}}{q-1} \\
& =1-\frac{j_{0}+j_{1}+\cdots+j_{a-1}}{a(p-1)} .
\end{aligned}
$$

If we take the strict inequalities, we have $\operatorname{ord}_{q}\left(g(\chi, \psi) \cdot g\left(\chi^{-1}, \psi\right)\right)>1$, which gives a contradiction to the identity $g(\chi, \psi) \cdot g\left(\chi^{-1}, \psi\right)=q \cdot \chi(-1)$.

6.2. The case $n=2$. We assume that $f_{1}, \ldots, f_{r}$ are homogeneous polynomials in $\mathbb{F}_{q}[x, z]$. The $f_{j}$ will satisfy the conditions of a normal crossing divisor if each $f_{j}$ can be factored into non-repeated linear factors over $\overline{\mathbb{F}}_{q}$ and also if the factors are unique to each $f_{j}$. The associated character sum is

$$
\sum_{(x, z) \in \mathbb{P}^{1}\left(\mathbb{F}_{q}\right)} \chi_{1}(f(x, z)) \ldots \chi_{r}\left(f_{r}(x, z)\right),
$$

where $\prod_{j=1}^{r} \chi_{j}^{d_{j}}=\chi_{0}$. In this case the Hilbert series of the top cohomology is given by $H_{\delta}(t)=(D-1)+(r-D-1) t$.

For example, if $r=4$, then there are three possible values for $D$, which are $D=1,2$ or 3 . If $D=1$, then $H_{\delta}(t)=2 t$ and the lower bound for the Newton polygon of $L(t)$ is given by the vertices $(0,0)$ and $(2,0)$.

If $D=2$, then $H_{\delta}(t)=1+t$ and the lower bound is given by the vertices $(0,0)$, $(1,0)$ and $(2,1)$.

In the last case where $D=3$, we have $H_{\delta}(t)=2$ and the lower bound is given by the vertices $(0,0)$ and $(2,0)$.

6.3. The case $n=3$. In this case the normal crossing divisor will be a collection of smooth curves in $\mathbb{P}^{2}\left(\mathbb{F}_{q}\right)$, having transversal intersections. For $f_{1}, \ldots, f_{r} \in$ $\mathbb{F}_{q}[x, y, z]$ the character sum

$$
\sum_{(x, y, z) \in \mathbb{P}^{2}} \chi_{1}\left(f_{1}(x, y, z)\right) \ldots \chi_{r}\left(f_{r}(x, y, z)\right)
$$

where $\prod \chi_{j}^{d_{j}}=\chi_{0}$, will produce a Hilbert series for $H^{r+3}\left(\Omega^{\bullet}\langle Y\rangle\right)^{(0)}$ of the form $H_{\delta}(t)=a_{0}+a_{1} t+a_{2} t^{2}$, where

$$
a_{0}=\frac{(D-1)(D-2)}{2}, \quad a_{1}=1-D^{2}+\sum_{j=1}^{r}\left(D d_{j}+\frac{1}{2} d_{j}\left(d_{j}-3\right)\right),
$$

and

$$
a_{2}=\frac{(D+1)(D+2)}{2}+\sum_{j=1}^{r}\left(\frac{1}{2} d_{j}\left(d_{j}-3\right)-D d_{j}\right)+\sum_{1 \leq j_{1}<j_{2} \leq r} d_{j_{1}} d_{j_{2}} .
$$


In order to illustrate the effect of the original choice of multiplicative characters, we will set $q=81$ and $r=3$. We first take $\delta_{j}=\delta_{j}^{\prime}$ for each $j$, and in the second case we suppose that $\delta j \neq \delta_{j}^{\prime}$ but $\delta_{j}=\delta_{j}^{\prime \prime}$.

Taking the quadratic character $\chi$ on $\mathbb{F}_{81}$ and setting $\chi=\chi_{j}$ for each $j$ will satisfy the conditions of the first case. If we assume for this example that $d_{j}=2$ for each $j$ the quadratic character also satisfies the necessary condition that $\prod_{j=1}^{3} \chi_{j}^{2}=\chi_{0}$. Therefore setting each $\delta_{j}=(q-1) / 2$ it follows that $D=3$. This results in the polynomial $(1-t)(1-q t)^{7}\left(1-q^{2} t\right)$. This polynomial has Newton polygon defined by the vertices $(0,0),(1,0),(8,7)$ and $(9,9)$

No consider the multiplicative characters

$$
\chi_{1}=\widehat{\chi}^{30}, \quad \chi_{2}=\widehat{\chi}^{60}, \text { and } \chi_{3}=\widehat{\chi}^{30} \text {. }
$$

Computing the 3 -tuples $\delta^{(I)}$ for $I=0,1,2,3$ gives that $\delta^{(0)}=\delta^{(2)}=(30,60,30)$ and $\delta^{(1)}=\delta^{(3)}=(10,20,10)$. The first set of values $(30,60,30)$ gives $D=3$ and the set $(10,20,10)$ gives $D=1$. For either set we have $H_{\delta}(1)=9$, which is the degree of the associated $L$-function. Computing the two Hilbert series gives

$$
H_{\delta^{(0)}}(t)=1+7 t+t^{2} \quad \text { and } \quad H_{\delta^{(1)}}(t)=3 t+6 t^{2} .
$$

The result is the polynomial $\left(1-q^{\frac{1}{2}} t\right)(1-q t)^{2}\left(1-q^{\frac{3}{2}} t\right)^{5}\left(1-q^{2} t\right)$ whose Newton polygon is determined by the vertices $(0,0),(1,1 / 2),(3,5 / 2),(8,10)$, and $(9,12)$.

6.4. Rational functions. One can also consider the case of a rational function $\mathrm{f} / \mathrm{g}$ using the character sum $\sum_{x \in \mathbb{P}^{2}} \chi_{1}(f(x)) \chi_{2}(g(x))$, where $\chi_{2}=\chi_{1}^{-1}$. If we require that $\operatorname{deg} f=\operatorname{deg} g=d$, then $D=d$ and the Hilbert series is

$$
H_{\delta}(t)=\frac{(d-1)(d-2)}{2}+(d-1)(2 d-1) t+\frac{(d-1)(d-2)}{2} t^{2} .
$$

We compute a few for small values of $d$. If $d=2$, then $H_{\delta}(1)=3$ and $H_{\delta}(t)=3 t$. This gives a lower bound for the Newton polygon of the $L$-function of $(0,0)$ and $(3,3)$, which has only one segment of length 3 and slope 1 .

If $d=3$, then $H(1)=12$ and $H(t)=1+10 t+t^{2}$, which results in the polynomial $(1-t)(1-q t)^{10}\left(1-q^{2} t\right)$. The lower bound is then given by a polygon that has one segment of length 1 and slope 0 , a segment of length 10 and slope 1, and a segment of length 1 and slope 2 defined by the vertices $(0,0),(1,0),(11,10),(12,12)$.

\section{REFERENCES}

[1] Adolphson, A. On the Dwork trace formula, Pacific Journal of Mathematics, 113 (1984) 257-268. MR749535 (85j:11178)

[2] Adolphson, A. And Sperber, S., Character sums in finite fields, Compositio Mathematica 52 (1984) 325-354. MR756726 (86c:11060)

[3] Adolphson, A. And Sperber, S., On twisted exponential sums, Mathematische Annalen, 290, (1991) 713-726. MR.1119948 (92g:11086)

[4] Adolphson, A. And Sperber, S., Exponential sums on $\mathbb{A}^{n}$, Israel Journal of Mathematics, 120 (2000) 3-21. MR.1815368 (2002g:11123)

[5] Adolphson, A. And Sperber, S., On the Jacobian ring of a complete intersection, Journal of Algebra, 304 (2006), 1193-1227. MR2265512 (2007g:13021)

[6] Adolphson, A. And Sperber, S., On the zeta function of a projective complete intersection, Illinois J. Math. 52 (2008), 389-417. MR2524643 (2010i:11139)

[7] Davenport, H., On character sums in finite fields, Acta Math. 71 (1939) 99-121. $\operatorname{MR} 0000252(1: 41 \mathrm{e})$

[8] DwORK, B., On the rationality of the zeta function of an algebraic variety, American Journal of Mathematics 82 (1960) 631-648. MR0140494 (25:3914) 
[9] Dwork, B., On the zeta function of a hypersurface, Publ. Math IHES Paris, 12 (1962) 5-68. MR0159823 (28:3039)

[10] Dwork, B., On the zeta function of a hypersurface II, Annals of Mathematics, 80 (1964) 227-299. MR0188215 (32:5654)

[11] KATZ, N., Estimates for nonsingular multiplicative character sums, International Mathematics Research Notices, 7 (2002) 333-349. MR1883179 (2003a:11106)

[12] KITA, M., On vanishing of the twisted rational de Rham cohomology assosciated with hypergeometric functions, Nagoya Math J. 135 (1994) 55-58. MR1295817 (95j:33043)

[13] Koblitz, N., p-adic Numbers, p-adic Analysis, and Zeta-Functions, Springer-Verlag, 1984. MR754003 (86c:11086)

[14] Monsky, P., p-Adic analysis and zeta functions, Lectures in Mathematics, Kyoto University Press, Tokyo, 1970. MR0282981 (44:215)

[15] RobbA, P., Une introduction naïve aux cohomologies de Dwork, Mémoire de la Société Mathématique de France no. 23114 (1986) 61-105. MR865812 (88a:14023)

[16] Saito, K., On a generalization of the de-Rham lemma, Annales de l'Institut Fourier, 26 no. 2, (1976) 165-170. MR0413155 (54:1276)

[17] Serre, J.P., Endomorphismes completement continus des espaces de Banach p-adiques, Publ. Math. I.H.E.S. 12 (1962), 69-85. MR0144186(26:1733)

LA PAZ, Bolivia

E-mail address: dollarhi@hotmail.com 